\title{
Equilibria in a Dynamic Global Game: The role of cohort effects *
}

\author{
Paul Heidhues ${ }^{\dagger}$ \\ WZB
}

\author{
Nicolas Melissas $\ddagger^{\ddagger}$ \\ University of Leicester
}

November 1, 2002

\begin{abstract}
We introduce strategic waiting in a global game setting. Players can wait in order to take a better informed decision. We allow for cohort effects, which naturally arise if the network externality in a given period depends on the mass of players who are actively using the technology at this point in time. Formally, cohort effects lead to intra-period network effects being greater than inter-period network effects. In the absence of cohort effects, our model has a unique rationalizable equilibrium. Cohort effects, however, can lead to multiple equilibria within the class of symmetric switching strategies.
\end{abstract}

JEL-codes: C72, C73, D82, D83.

Keywords: Global game, Strategic Waiting, Coordination, Strategic Complementarities, Period-specific Network Effects.

\footnotetext{
*We want to thank Frank Heinemann, Christian Hellwig, Larry Karp, In Ho Lee, and Robin Mason for helpful discussions. We started to work on this paper during the Summer Symposium in Economic Theory in Gerzensee, 2000. We hereby thank them for providing us with a very research-friendly atmosphere. We would also like to thank seminar participants at the Free University Berlin and especially Helmut Bester, Instituto d'Analisis Economico (CSIC) and the University of Pittsburgh for comments. We gratefully acknowledge financial assistance provided by the European Commission through its TMR program (Contract number FMRX-CT98-0203). This version is still very preliminary.

${ }^{\dagger}$ Wissenschaftszentrum Berlin für Sozialforschung (WZB). Correspondence can be sent to Paul Heidhues (heidhues@wz-berlin.de), WZB, Reichpietschufer 50, 10785 Berlin, Germany.

${ }_{\ddagger}^{\ddagger}$ Correspondence can be sent to Nicolas Melissas (ndm9@leicester.ac.uk), University of Leicester, University Road, Leicester, LE1 7RH, UK.
} 


\section{Introduction}

In many economic situations, the optimal action of an economic agent is complementary to the actions undertaken by other agents. For example, a consumer's payoff from buying a computer software typically increases as the number of consumers who use this technology increases. One reason for this is a direct network effect. The amount of people with whom the consumer can exchange relevant data increases. Another is an indirect network effect. The more popular the software, the more likely complementary products are offered. Such indirect network effects are common. Consider a consumer deciding on whether or not to buy a video recorder (CD player or DVD player). As there are more users, more videos (CD's or DVD's) will become available to the consumer. Or, think of a consumer who decides to buy a durable consumption good such as a car. As more consumers buy this brand of car, more repair shops will have the know-how and spare parts to repair the car quickly. Complementarity of optimal actions is also a key ingredient of Diamond and Dybvig's (1983) famous bank-run model in which a player's payoff from withdrawing depends on the actions undertaken by the other players. Similarly, in Obstfeld's (1996) model of currency crises a speculative attack is only successful if launched by a sufficiently high number of traders. Models of situations in which the agents' optimal actions are complementary to each other are often plagued by multiple equilibria with self-fullfilling beliefs: If a player expects the other players to take the action (i.e withdraw in Diamond and Dybvig's model), then it's in her best interest to withdraw as well. If a player expects the other players not to withdraw, she wants to refrain from withdrawing. This multiplicity result is annoying from an economic-policy point of view. Without an adequate theory of equilibrium selection, one cannot use these theories to predict the stability of a financial system or of a currency peg nor can one predict the market outcome in markets with network effects. How then does one judge, for example, whether costly policies to enhance the stability of the financial system should be implemented?

For two-player coordination games, Carlsson and van Damme (1993), henceforth $\mathrm{CD}$, developed an equilibrium selection theory, which was adopted to (a specific) coordination problem with a continuum of players by Morris and Shin (1998). CD assume that the agents' payoffs depend on the action chosen by the other agent in the economy and some unknown economic fundamental summarized by the state of the world $\theta$. Agents receive different signals about $\theta$, which generate beliefs 
about the state of the world and a hierarchy of higher order beliefs (beliefs about the other agent's beliefs, beliefs about the other agent's beliefs about his beliefs, etc...). ${ }^{1} \mathrm{CD}$ called this incomplete information game a global game and showed that if the potential type space is rich enough, the game has a unique equilibrium.

Thus, the global game framework enables researchers to base policy recommendations on theory that predicts behavior in coordination games (e.g the probability of a bank run or of a speculative currency attack). It has been used to model currency crises (Morris and Shin (1998), Corsetti and al. (2000), ...), bank runs (Goldstein and Pauzner (2000), Rochet and Vives (2000),...) and car-dealer markets (Dönges and Heinemann (2000)). All these papers are based on static models. In reality, however, many economic coordination problems are essentially dynamic. Players can always postpone their investment decisions (or their withdrawing decision) in order to make a more informed decision at a later point in time. What happens in a coordination game with incomplete information once we allow for strategic waiting? To better understand this question let us first explain the logic underlying any global game.

Consider a continuum of players who have the opportunity to undertake a risky investment, whose return depends positively on the realization of a random variable and on the mass of investors. All players possess some noisy private information concerning the realization of the random variable. Players who received a "very high" signal always invest, since they believe that the state of the world is so good that investing is always profitable - independent of the actions undertaken by the other players. Consider now a player that possess a "high" but not a "very high" signal. If she expects no other player to invest, then she would rather refrain from investing. She knows, however, that all players with a very high signal invest. Given her signal, it is equally likely that the other players received a higher or lower signal than herself. Therefore, in equilibrium, she cannot expect that no other player invests. As her signal is "high," her knowledge that everyone with a very high signal invests is enough to induce her to invest as well. This will, in turn, convince other players possessing a signal a little less favorable then hers to also

\footnotetext{
${ }^{1}$ Carlsson and van Damme's work is based on the insight developed in Rubinstein's (1989) famous electronic mail game, in which he illustrated that the risk-dominated equilibrium of the common knowledge game is selected as the unique equilibrium in the absence of common knowledge. That heterogeneity of agents can lead to a unique equilibrium in situations in which the agents actions are complementary to each other was shown earlier by Postlewaite and Vives (1987) in a bank-run model.
} 
invest, etc... . This process of iterative elimination of dominated strategies stops at the point where a player, who believes that everyone with a lower signal than herself does not invest, is indifferent between investing and not investing. Similar, because it is a dominant strategy not to invest for players with "very low" signals, players with low signals refrain from investing. Iteratively eliminating players for whom it is a dominant strategy not to invest, there is a critical player who is indifferent between investing and not investing if she believes that all players with a higher signal than herself invest. With a uniform prior, the two iterative processes stop at the same point and, hence, there is a unique equilibrium. This equilibrium is characterized by a critical treshold, below which no player invests and above which all players invest.

It is not clear, however, whether this line of reasoning can be extended to a dynamic setting. In a dynamic setting, we would expect the most "extreme" players (e.g. those players possessing a "very high" signal) to move first. Thus, the period-two distribution of signals is a truncated one (and this is common knowledge). Given that the "very high" types have invested at time one, do the "high" types then still have a strong incentive to invest at time two, or do their investment incentives depend on their expectations (of the other players' actions at time two)?

Furthermore, in many dynamic settings the benefit of moving early depends not only on the aggregate mass of investors but also on when other players invest. Consider, for example, a consumer buying a computer software early on. In the interim period in which late movers have not invested yet, this early mover is subject to a network effect depending on the mass of early movers only. Here, the intra-period network effects between early investors are greater than the inter-period network effects with late movers. We call this an early mover cohort effect. Similar, if the intra-period network effects between second-period investors are greater than the inter-period ones with first-period investors, we say that there is a late mover cohort effect. $^{2}$ Cohort effects yield an additional coordination aspect: when to invest. To understand why cohort effects may matter, suppose that the inter-period network effects are zero. Then one would expect an equilibrium to exist in which all players invest in the first period and one in which all players invest in the second period. That is, one would expect multiple equilibria to exist. This raises the question:

\footnotetext{
${ }^{2}$ In Section 3, we point out that both type of cohorts effects occur in dynamic situations in which the network externality in a given period depends on the mass of players that are currently active.
} 
Under what conditions do multiple equilibria exist with less extreme cohort effects?

To investigate the dynamic extension, we analyze a set-up identical to the one we described above, with the exception that players can invest in either of two periods. If a player waits, she gets a more informative signal (concerning the realization of the random variable) at the cost of foregone profits. Our model is simple in the sense that we assume a uniform distribution of period-one signals. Moreover, we assume that each player at time two can either receive good or bad news and that the second-period signal constitutes a sufficient statistic for a player's beliefs about the random variable. This set of assumptions highly simplify the computation of our equilibrium strategies and permit us to get closed-form solutions. This enables us to investigate the role of cohort effects. We characterize for which parameter values our model has multiple equilibria in symmetric switching strategies and show that for some underlying economic interpretations our model exhibits multiple equilibria while for others it does not. In particular, in the absence of cohort effects, our model has a unique equilibrium in rationalizable strategies.

This is not the first paper to introduce dynamic elements in a global game. Morris and Shin (1999) and Chamley (1999) studied a dynamic global game in which the state of the world evolves stochastically through time. In each period all players face a new investment opportunity and players observe a statistic correlated with past realizations of the state of the world. In those models players have no incentives to wait and act later on the basis of more information. Under some additional assumptions, the authors show that each period can be analyzed as a static global game. Thus, their models have a unique equilibrium. ${ }^{3}$

To the best of our knowledge, only Dasgupta (2001) introduced elements of strategic waiting in a global game. Players can invest in two periods. If a player delays, she observes a noisy signal about the past economic activity (at the cost of foregone profits). Dasgupta shows that his game, under some additional assumptions on the prior distribution and the signalling technology, is characterized by a unique equilibrium in the class of switching strategies. The main difference between our paper and Dasgupta (2001) is that we investigate cohort effects, which are not present in his model in which payoffs depend only on the aggregate number of investors. Another difference is that in his model one wants to delay to engage

\footnotetext{
${ }^{3}$ Frankel and Pauzner (2000) investigate a dynamic model in which investment opportunities arrive at different point in time. ...
} 
in social learning, while in our model a player delays to obtain a more precise signal.

The remainder of this paper is organized as follows. In section 2, we introduce our formal model. In section 3 , we relate the parameters of our model to different economic environments. In section 4 , we analyze the model and characterize the set of parameter values for which it has multiple equilibria. Final comments are summarized in the fifth and final section. Appendix 1 derives the number of investors as function of the realized fundamental and a given strategy profile. Some more technical proofs are relegated to Appendices 2, 3, and 4.

\section{The model}

Assume a continuum of risk-neutral players with mass one that are indexed along the line $[0,1]$. All players have the opportunity to undertake one risky investment project. Investments are irreversible. A player can invest at time one, at time two, or can decide not to invest at all. If player $i$ decides to invest at time one, she gets a utility $U_{1}^{i}$ equal to:

$$
U_{1}^{i}=\theta+n_{1}+\alpha n_{2}-1,
$$

where $n_{1}\left(n_{2}\right)$ denotes the mass of players who invest at time one (two). The state of the world $\theta$ is randomly drawn from a uniform distribution along the entire real line. A period-two investor enjoys a utility equal to:

$$
U_{2}^{i}=\tau\left(\theta+\gamma n_{1}+n_{2}-1-\Delta\right) .
$$

If player $i$ decides not to invest in any of the two periods, she gets zero. Throughout, we assume that $\tau, \alpha, \gamma \in[0,1]$ and that $\Delta \geq 0$. We postpone the discussion of the economic motivation for our payoff structure until the next section.

All players possess a private and imperfect signal concerning the realized state of the world. Formally, player $i$ 's first-period signal, $s_{1}^{i}$, equals:

$$
s_{1}^{i}=\theta+\epsilon_{2}^{i}+\epsilon_{1}^{i},
$$

where $\epsilon_{2}^{i} \sim U[-\epsilon, \epsilon]$ and $\epsilon_{1}^{i} \in\{-\epsilon, \epsilon\}$. The prior probability that $\epsilon_{1}^{i}=-\epsilon$ equals $\frac{1}{2}$. $\epsilon_{2}^{i}$ and $\epsilon_{1}^{i}$ are independently distributed. Player $i$ 's second-period signal, $s_{2}^{i}$, equals:

$$
s_{2}^{i}=\theta+\epsilon_{2}^{i} .
$$


The first- (and second-) period errors $\epsilon_{1}^{i}\left(\epsilon_{2}^{i}\right)$ are uncorrelated among the different players.

Note that our model possesses some "desirable" features that highly simplify the computation of our equilibrium strategies and enable a direct comparison with the static counterparts of our model. First, note that $s_{1}^{i}$ is constructed by adding noise to $s_{2}^{i}$. In statistical terms, this means that $s_{2}^{i}$ is a sufficient statistic for $s_{1}^{i}$. In particular, this implies that $E\left(\theta \mid s_{2}^{i}, s_{1}^{i}\right)=E\left(\theta \mid s_{2}^{i}\right)$. Second, we know that $s_{2}^{i}=\theta+\epsilon_{2}^{i}$. This last equality can be rewritten as $\theta=s_{2}^{i}-\epsilon_{2}^{i}$. Hence, $\theta \mid s_{2}^{i} \sim U\left[s_{2}^{i}-\epsilon, s_{2}^{i}+\epsilon\right]$, and $E\left(\theta \mid s_{2}^{i}\right)=s_{2}^{i}$. Similar, one has $\theta \mid s_{1}^{i} \sim U\left[s_{1}^{i}-2 \epsilon, s_{1}^{i}+2 \epsilon\right]$, and $E\left(\theta \mid s_{1}^{i}\right)=s_{1}^{i}$. That is the errors are uniformly distributed in both periods. This ensures that if players were only allowed to invest either in the first or in the second period, then our game would be characterized by a unique equilibrium in switching strategies. ${ }^{4}$

To avoid confusion, we detail the timing of the game we study.

0 ) Nature chooses $\theta$. All players receive their first-period signals.

1) All players simultaneously decide whether to invest or wait.

2) Player $i$ observes whether $\epsilon_{1}^{i}=\epsilon$ or $\epsilon_{1}^{i}=-\epsilon$ but not $n_{1}$. If she did not invest at time one, she decides whether or not to do so at time two.

3) All players receive their payoffs and the game ends.

Each player's action space $A=$ \{invest, not invest $\}$. Player $i$ 's observable history at time one is $H_{1}=\left\{s_{1}^{i} \mid s_{1}^{i} \in \Re\right\}$ and at time two it is $H_{2}=\left\{\left(s_{1}^{i}, s_{2}^{i}\right) \mid s_{1}^{i} \in \Re \wedge s_{2}^{i} \in\right.$ $\left.\left\{s_{1}^{i}-\epsilon, s_{1}^{i}+\epsilon\right\}\right\}$. Let $H=H_{1} \cup H_{2}$. A symmetric (pure) strategy of player $i$ is a (measurable) function $\sigma: H \rightarrow A$, with the interpretation that $\sigma\left(s_{1}^{i}\right)$ represents the action taken by player $i$ at time one given her first period signal. $\sigma\left(\left(s_{1}^{i}, s_{2}^{i}\right)\right)$ represents the action taken by player $i$ at time two given $\left(s_{1}^{i}, s_{2}^{i}\right)$. By assumption player $i$ can only invest once. Therefore we impose the following restriction on $\sigma$ : If $\sigma\left(s_{1}^{i}\right)=$ invest, then $\sigma\left(\left(s_{1}^{i}, \cdot\right)\right)=$ not invest. Throughout this paper, we focus on symmetric switching strategies; that is strategies that can be parameterized by a vector $k \equiv\left(k_{1}, k_{2}\right)$ with the interpretation that:

(i) $\sigma\left(s_{1}^{i}\right)=$ invest if and only if $s_{1}^{i}>k_{1}$,

(ii) $\sigma\left(\left(s_{1}^{i}, s_{2}^{i}\right)\right)=$ invest if and only if $s_{2}^{i}>k_{2}$ and player $i$ did not invest in period 1 .

An equilibrium in symmetric switching strategies is a $k^{*}$ such that player $i$ 's strat-

\footnotetext{
${ }^{4}$ Using a similar argument to Morris and Shin (2001), this extends to an essentially unique equilibrium in rationalizable strategies.
} 
egy is a best response at every information set given (i) his beliefs about the state of the world, and given (ii) the equilibrium behavior of all other agents.

\section{Economic Interpretations}

In this section, we show that the payoff structure of our model accommodates a wide variety of more specific models.

Fixed Horizon Technology Adoption Problem (=FHTAP). Suppose players can invest in a new technology with an unknown quality. This technology exhibits positive network effects and becomes obsolete in period $T+1$. For simplicity, players are only allowed to invest in period 1 or period 2 and have a common discount factor $\delta$. Call a player who invested at time one (two) an (a) early (late) adopter. When investing, players need to pay a setup cost $s \geq 0$. The (net of any per-period cost) return of the investment in period $t(t=1, \ldots, T)$, is given by $v_{t}^{i}=\tilde{\theta}+m_{t}$, where $m_{t}$ denotes the mass of players who invest in period $t$ or who have invested earlier. Assume, for the sake of simplicity, that $T=2$. In this case the payoff of a player investing in period 1 is given by

$$
V_{1}^{i}=(1+\delta) \tilde{\theta}+(1+\delta) n_{1}+\delta n_{2}-s
$$

and of a player investing in period 2 is given by

$$
V_{2}^{i}=\delta\left(\tilde{\theta}+n_{1}+n_{2}\right)-\delta s
$$

Setting $\theta=\tilde{\theta}-\frac{s}{(1+\delta)}+1$ and using the following utility transformation $U_{t}^{i}=\frac{V_{t}^{i}}{(1+\delta)}$ shows that this economic model is a special case of our model in which $\alpha=\tau=$ $\frac{\delta}{1+\delta}<1, \gamma=1$, and $\Delta=\frac{\delta}{1+\delta} s^{5}$

Note that at time one the early adopters do not enjoy any network benefits from the late adopters. Therefore early adopters care more about the mass of players who bought the technology at time one than about the mass of players who bought it at time two (which explains why in this case $\alpha<1$ ). The FHTAP model can be interpreted as a stylized model of the credit card industry. The more popular a

\footnotetext{
${ }^{5}$ If $T>2$, one should set $\theta=\tilde{\theta}-\frac{s}{1+\delta+\ldots+\delta^{T-1}}+1$ and use the following utility transformation: $U_{t}^{i}=\frac{V_{t}^{i}}{1+\delta+\ldots+\delta^{T-1}}$. After some computations we then get that $\alpha=\tau=\frac{\delta+\ldots+\delta^{T-1}}{1+\delta+\ldots+\delta^{T-1}}, \gamma=1$, and $\Delta=\frac{\delta^{T} s}{\left(\delta+\ldots+\delta^{T-1}\right)\left(1+\delta+\ldots+\delta^{T-1}\right)}$.
} 
credit card becomes, the more widespread its acceptance will be. Early adopters of a credit card are therefore hampered by its small installed base in the sense that they will find few shops willing to accept it. On the other hand, late movers care as much about the mass of early as about the mass of late adopters (which explains why in this case $\gamma=1) .{ }^{6}$ Whenever an early (late) adopter exhibits a stronger preference for her fellow adopters to invest early (late), we say that our model exhibits a cohort effect for the early (late) adopters.

Adopting a Technology with a Fixed Lifespan (=ATFL). Rather than assuming that the technology becomes obsolete at time $T+1$, suppose the technology, once bought, can be used for $T$ periods. For the sake of simplicity, assume that $T=2$ (i.e. in this case an early adopter uses her technology at times one and two, while a late adopter uses it at times two and three). If the setup remains otherwise unchanged, one can use a similar procedure as above to show that this is a special case of our model in which $\tau=\delta, \alpha=\delta \gamma<\gamma=\frac{1}{1+\delta}<1$, and $\Delta=0 .{ }^{7}$

To illustrate this interpretation, consider the following example: Assume everyone has the opportunity to buy a video player. The more people who buy a video player, the higher the availability of video movies, video rental stores, etc. A video player can only be used for two periods. Everyone knows that at time 3 the DVD player will be introduced in our economy. As DVD technology is superior to video technology, from time 3 on, no one wants to buy a new video player anymore. However, people only switch to the superior DVD technology once their video player becomes "too old" (i.e. early adopters switch to the superior technology at time three, while late adopters switch to the new technology at time four). In this set-up for the same reason as the one explained in our earlier interpretation, our model exhibits a cohort effect for the early adopters. However, in this case late adopters know that the installed base will become smaller at time three due to the early adopters' switching to the new technology. Therefore, the ATFL model also exhibits a cohort effect for late adopters.

Technology Adoption Problem with Partially Compatible Technologies (=TAPPCT). Consider the same setup as in the Fixed Horizon Technology Adoption Problem

\footnotetext{
${ }^{6}$ In the credit card example, one may want to think about $T$ as tending towards infinity.

${ }^{7}$ If $T>2$ one must apply the utility transformation which appears in our earlier footnote. One can check that the values of $\tau, \alpha$ and $\Delta$ then remain unchanged. $\gamma$ would then be equal to $\frac{1+\delta+\ldots+\delta^{T-2}}{1+\delta+\ldots .+\delta^{T-1}}$.
} 
(with $T=2$ ) except for the following two changes. First, assume that the early adopters get the following per-period payoffs: $v_{11}^{i}=\tilde{\theta}+n_{1}$ and $v_{21}^{i}=\tilde{\theta}+n_{1}+\tilde{\alpha} n_{2}$ (where $v_{t 1}^{i}$ denotes the payoff received by player $i$ at time $t(t=1,2)$ given that she adopted the new technology at time one and where $\tilde{\alpha} \in[0,1))$. Second, assume late adopters get the following second-period payoff: $v_{22}^{i}=\tilde{\theta}+\tilde{\gamma} n_{1}+n_{2}$ (where $\tilde{\gamma} \in[0,1))$. In this case the present value of adopting early equals

$$
V_{1}^{i}=(1+\delta) \tilde{\theta}+(1+\delta) n_{1}+\delta \tilde{\alpha} n_{2}-s,
$$

while a late adopter receives

$$
V_{2}^{i}=\delta \tilde{\theta}+\delta \tilde{\gamma} n_{1}+\delta n_{2}-\delta s
$$

Applying the same utility transformation to these payoffs as the one explained in our FHTAP-interpretation, one can check that this interpretation is a special case of our model in which $\alpha=\tilde{\alpha} \frac{\delta}{1+\delta} \in[0,1), \gamma=\tilde{\gamma}, \tau=\frac{\delta}{1+\delta}$ and $\Delta=\frac{\delta}{1+\delta} s$.

For example, one can think of our players as consumers who choose whether or not to buy a computer. Early adopters buy a computer equipped with Windows 3.11. Between time one and time two, the seller introduces a superior computer equipped with Windows 95. As both operating systems are different, a late adopter can not always be helped by an early adopter whenever she faces a problem (and vice versa). Therefore, in this example, there is a cohort effect for both the early and the late adopters.

Pledging to Invest with Early Mover Advantage (=PIEMA). Suppose there are two periods in which players can commit to invest into a project prior to the time in which the project will take place. For example, firms may commit to buy some land in a soon-to-be developed industrial zone (or individuals may commit to become a member of some club or lobbying organization). In the first period, the land is sold at a lower price than in the second period (or there is a reduced membership rate). The more players invest in either period, the better the infrastructure provided (or the more exciting it will be to visit the club or the more influential will the lobbying organization be). In period 3, all players that committed to invest pay the amount due and start getting the benefit from the planned activity. This can be captured by a model in which $\alpha=\gamma=\tau=1$ and $\Delta>0$. This example is thus void of any cohort effects. 


\section{Analysis of Strategic Waiting}

In this section, we first characterize the equilibria within the class of symmetric switching strategies. Our characterization shows that cohort effects can lead to multiple equilibria. We then prove that in the absence of cohort effects, there exists an essentially unique equilibrium in rationalizable strategies. We furthermore discuss under what parameter conditions our model has a unique equilibrium in class of symmetric switching strategies.

To solve the dynamic game, we show that every symmetric equilibrium in switching strategies can be found by analyzing a system of equations. We start with some useful definitions. Let

$$
h\left(s_{2}^{i}, k\right) \equiv s_{2}^{i}+E\left(\gamma n_{1}+n_{2} \mid s_{2}^{i}, k\right)-1-\Delta .
$$

$h\left(s_{2}^{i}, k\right)$ is the expected payoff of a player who invests in the second period after getting a signal $s_{2}^{i}$, expecting that all other players play the strategy $k$. Similar, we define

$$
W\left(s_{1}^{i}, k\right) \equiv \frac{\tau}{2} \max \left\{0, h\left(s_{1}^{i}+\epsilon, k\right)\right\}+\frac{\tau}{2} \max \left\{0, h\left(s_{1}^{i}-\epsilon, k\right)\right\} .
$$

$W\left(s_{1}^{i}, k\right)$ the gain of waiting for a player $i$ with a signal $s_{1}^{i}$ who believes that all other players play $k$. If player $i$ postpones her investment decision, then with probability $1 / 2$ she will get "bad news," i.e. she will learn that at time one she was to optimistic because $\epsilon_{1}^{i}=+\epsilon$. With probability $1 / 2$, however, she will receive "good news" in the sense that she will learn that $\epsilon_{1}^{i}=-\epsilon$. Equation (2) states that player $i$ 's gain of waiting equals her expected second-period payoff given that she will take an optimal second-period investment decision (i.e. not invest at time two if and only if her gain of investing is negative). For brevity, define

$$
g\left(s_{1}^{i}, k\right) \equiv s_{1}^{i}+E\left(n_{1}+\alpha n_{2} \mid s_{i}^{1}, k\right)-1-W\left(s_{i}^{1}, k\right) .
$$

Trivially, it is optimal to invest in the first period for a player with a signal $s_{1}^{i}$ (who believes that all his rivals play $k$ ) if and only if $g\left(s_{1}^{i}, k\right) \geq 0$.

A necessary condition for a strategy profile $k *$ in which $k_{t}^{*}<\infty$ for $t+1,2$ to be an equilibrium (strategy profile) in symmetric switching strategies is that it satisfies the following two equations:

$$
g\left(k_{1}^{*}, k^{*}\right)=0,
$$




$$
h\left(k_{2}^{*}, k^{*}\right)=0 .
$$

Equation (4), which can be rewritten as

$$
k_{1}^{*}+E\left(n \mid k^{*}, s_{i}^{1}=k_{1}^{*}\right)-1=W\left(k, s_{i}^{1}=k_{1}^{*}\right),
$$

states that a player possessing a first-period signal $s_{1}^{i}=k_{1}^{*}$ must be indifferent between investing and waiting. Equation (5) says that a player who receives a second-period signal $s_{2}^{i}=k_{2}^{*}$ is indifferent between investing and not investing. In case $k_{1}^{*}=\infty$, equation (4) must be replaced by the condition $g\left(s_{1}^{i}, k^{*}\right) \leq 0$, for all $s_{1}^{i}$. That is it must be optimal to refrain from investing for all first period signals. Similar, in case $s_{2}^{i}=\infty$, condition (5) must be replaced by the condition $h\left(k_{2}^{*}, k^{*}\right) \leq 0$ for all $s_{2}^{i}$.

If $g\left(s_{1}^{i}, k\right)$ (respectively $h\left(s_{2}^{i}, k\right)$ ) are monotonically increasing in $s_{1}^{i}$ (respectively $s_{2}^{i}$ ), then any strategy profile $k^{*}$ satisfying (4) and (5) is clearly an equilibrium strategy profile. However, note that in general $h(\cdot)$ can be a function of $E\left(n_{2} \mid \cdot\right)$. In some symmetric switching equilibria, players refrain from investing for sufficiently low signals and all players invest immediately in the first period for sufficiently high signals. For intermediate signals, however, players wait and invest in the second period when receiving good news. In such a candidate equilibrium, $h(\cdot)$ need not be monotone in $s_{2}^{i}$ as $E\left(n_{2} \mid \cdot\right)$ is not. When characterizing the set of symmetric switching equilibria, we first look for candidate equilibria that solve equations (4) and (5) and then carefully verify that these candidate equilibria are indeed equilibria. To economize on notation, we will from now on denote equilibrium strategy profiles (and candidate equilibria) by $k$ rather than $k^{*}$.

We refer to an equilibrium $k$ in which no player invests in the second period as an immediate investment equilibrium. Formally, $k$ is an immediate investment equilibrium if and only if $k_{2} \geq k_{1}+\epsilon$.

Proposition 1 There exists an immediate investment equilibrium if and only if $\Delta \geq-\frac{1}{2}+\epsilon+\frac{3}{4} \gamma$. In an immediate investment equilibrium $k_{1}=\frac{1}{2}$.

Proof: In an immediate investment equilibrium no player invests in the second period. Hence,

$$
h\left(s_{2}^{i}, k\right)=s_{2}^{i}+\gamma E\left(n_{1} \mid s_{2}^{i}, k\right)-1-\Delta .
$$

As shown in Appendix 1, $E\left(n_{1} \mid s_{2}^{i}, k\right)$ is weakly increasing, and thus $h\left(s_{2}^{i}, k\right)$ is strictly increasing in an immediate investment equilibrium. By definition, we look 
for an equilibrium in which $h\left(s_{2}^{i}=k_{1}+\epsilon, k\right) \leq 0$. Hence, the gain of waiting must be equal to zero. Therefore, $k_{1}$ must be set such that a player who possesses a signal $s_{1}^{i}=k_{1}$ is indifferent between investing and not investing. Hence $k_{1}$ solves the following equation

$$
k_{1}+E\left(n_{1} \mid s_{1}^{i}=k_{1}, k\right)-1=0 .
$$

Using Appendix 1 and the fact that

$$
E\left(n_{1} \mid s_{1}^{i}=k_{1}, k\right)=\frac{1}{4 \epsilon} \int_{k_{1}-2 \epsilon}^{k_{1}+2 \epsilon} n_{1}(\theta, k) d \theta,
$$

it is easy to verify that $E\left(n_{1} \mid s_{1}^{i}=k_{1}, k\right)=\frac{1}{2}$. Thus, in an immediate investment equilibrium $k_{1}=\frac{1}{2}$. Using this fact to rewrite the condition that no player has an incentive to invest in the second period, i.e. that $h\left(s_{2}^{i}=k_{1}+\epsilon, k\right) \leq 0$, gives

$$
\frac{1}{2}+\epsilon+\gamma E\left(n_{1} \mid s_{2}^{i}=k_{1}+\epsilon, k\right) \leq 1+\Delta .
$$

Using Appendix 1 and the fact that

$$
E\left(n_{1} \mid s_{2}^{i}=k_{1}+\epsilon, k\right)=\frac{1}{2 \epsilon} \int_{k_{1}}^{k_{1}+2 \epsilon} n_{1}(\theta, k) d \theta,
$$

it is easy to verify that $E\left(n_{1} \mid s_{2}^{i}=k_{1}+\epsilon, k\right)=\frac{3}{4}$. Substituting this into equation (6) and rewriting yields $\Delta \geq-\frac{1}{2}+\epsilon+\frac{3}{4} \gamma$, which is a necessary condition for an immediate investment equilibrium to exist. Because we already established that $h\left(s_{2}^{i}, k\right)$ is strictly increasing, it suffices to show that $g(\cdot)$ is (weakly) increasing to show that an immediate investment equilibrium exists whenever $\Delta \geq-\frac{1}{2}+\epsilon+\frac{3}{4} \gamma$. First, observe that for all $s_{1}^{i}<k_{2}-\epsilon$, one has

$$
g\left(s_{1}^{i}, k\right)=s_{1}^{i}+E\left(n_{1} \mid s_{1}^{i}, k\right)-1,
$$

which is strictly increasing in $s_{1}^{i}$ because $E\left(n_{1} \mid s_{1}^{i}, k\right)$ is weakly increasing in $s_{1}^{i}$. Second, for all $k_{2}-\epsilon<s_{1}^{i}<k_{2}+\epsilon$,

$$
g\left(s_{1}^{i}, k\right)=s_{1}^{i}+E\left(n_{1} \mid s_{1}^{i}, k\right)-1-\frac{\tau}{2} h\left(s_{1}^{i}+\epsilon, k\right) .
$$

Using Lemma (3), one can rewrite this equation as

$g\left(s_{1}^{i}, k\right)=\left(1-\frac{\tau}{2}\right) s_{1}^{i}+\frac{1}{2}\left[E\left(n_{1} \mid s_{2}^{i}=s_{1}^{i}-\epsilon, k\right)+(1-\tau \gamma) E\left(n_{1} \mid s_{2}^{i}=s_{1}^{i}+\epsilon, k\right)-\tau \epsilon-(2-\tau)+\tau \Delta\right]$. 
Since $E\left(n_{1} \mid s_{2}^{i}=s_{1}^{i}-\epsilon, k\right)$ and $E\left(n_{1} \mid s_{2}^{i}=s_{1}^{i}+\epsilon, k\right)$ are weakly increasing in $s_{1}^{i}$, and $\tau, \gamma \leq 1, g\left(s_{1}^{i}, k\right)$ is strictly increasing in $s_{1}^{i}$ in this subcase. Third, for all $k_{2}+\epsilon<s_{1}^{i}$, one has

$$
g\left(s_{1}^{i}, k\right)=s_{1}^{i}+E\left(n_{1} \mid s_{1}^{i}, k\right)-1-\frac{\tau}{2}\left[h\left(s_{1}^{i}-\epsilon, k\right)+h\left(s_{1}^{i}+\epsilon, k\right)\right] .
$$

Rewriting this equation using Lemma (3) yields

$g\left(s_{1}^{i}, k\right)=(1-\tau) s_{1}^{i}+\frac{(1-\tau \gamma)}{2}\left[E\left(n_{1} \mid s_{2}^{i}=s_{1}^{i}-\epsilon\right)+E\left(n_{1} \mid s_{2}^{i}=s_{1}^{i}+\epsilon\right)\right]-(1-\tau)+\tau \Delta$.

Since $E\left(n_{1} \mid s_{2}^{i}=s_{1}^{i}-\epsilon\right)$ and $E\left(n_{1} \mid s_{2}^{i}=s_{1}^{i}+\epsilon\right)$ are weakly increasing in $s_{1}^{i}$, and $\tau, \gamma \leq 1, g\left(s_{1}^{i}, k\right)$ is weakly increasing in $s_{1}^{i}$ in this subcase. Q.E.D.

The parameter condition under which an immediate investment equilibrium exists is intuitive. As the payoff reduction for late movers $\Delta$ increases, players have an incentive to move early and thus an immediate investment equilibrium is more likely to exist. As $\gamma$ decreases, a player who deviates in order to invest late enjoys a smaller (inter-period) network effect, which makes deviating less attractive. Hence, as $\gamma$ decreases, an immediate investment equilibrium is more likely to exists. To understand why an increase in $\epsilon$ makes it harder to sustain an immediate investment equilibrium, consider a player with a signal $s_{1}^{i}=1 / 2$. This player is uncertain about whether the fundamental $\theta$ is high enough to make his investment profitable. As $\epsilon$ increases, more uncertainty about $\theta$ is resolved between period one and two, which makes it more desirable to wait in order to receive more information.

To further understand the role of $\epsilon$, it is useful to note that the expected network benefit for a player with a signal $s_{1}^{i}$ is $1 / 2$ in an immediate investment equilibrium. Formally, using Appendix 1 and the fact that

$$
E\left(n_{1} \mid s_{1}^{i}=k_{1}, k\right)=\frac{1}{4 \epsilon} \int_{k_{1}-2 \epsilon}^{k_{1}+2 \epsilon} n_{1}(\theta, k) d \theta,
$$

it is easy to verify that $E\left(n_{1} \mid s_{1}^{i}=k_{1}, k\right)=\frac{1}{2}$. Intuitively, player $i$ knows that all players possessing a signal higher (lower) than hers invest (do not invest) at time one. Player $i$ asks herself the question: What is the mass of players who received a first-period signal greater than $k_{1}$ ? Player $i$ knows that $\theta$ lies in a $2 \epsilon$ neighborhood of $s_{1}^{i}$. If $\theta>s_{1}^{i}$, she knows that more than $1 / 2$ of the population possesses a signal higher than hers. Conversely, if $\theta<s_{1}^{i}$, she knows that more than $1 / 2$ of the population posses a signal strictly lower than hers. Given hat $\theta \mid s_{1}^{i}$ 
is symmetrically distributed around $s_{1}^{i}$, player $i$ knows that the event $\theta>s_{1}^{i}$ is as likely to occur as the event $\theta<s_{1}^{i}$. Therefore $E\left(n_{1} \mid s_{1}^{i}=k_{1},\left(k_{1}, \infty\right)\right)=1 / 2$. Stated differently, player $i$ always believes to lie in the center of the world. She always expects half of the population to possess a signal strictly higher than hers, with the other half possessing a signal strictly lower than hers.

Now, for simplicity, suppose that there is no late mover cohort effect $\gamma=0$ and that $\Delta=0$. Then an immediate investment equilibrium does not exist whenever $\epsilon>1 / 2$. The intuition for this result is as follows: In an immediate investment equilibrium a player with a signal $s_{1}^{i}=k_{1}$ is indifferent between investing and not investing, which is the action she will take if she decides to wait. So her expected payoff must be zero. Furthermore, as discussed above he expects half of the population to get a better signal than herself. So her expected gain from the network effect is $1 / 2$. But if $\epsilon>1 / 2$, this player could wait, forfeit the expected network effect and only invest if she learns that she was to pessimistic. In this case her expected payoff when getting good news changes by $\epsilon-1 / 2$, while her expected payoff when getting bad news remains zero. So if $\epsilon>1 / 2$ this is a profitable deviation and an immediate investment equilibrium cannot exist.

We refer to an equilibrium $\left(\infty, k_{2}\right)$ in which no player invests in the first period as a complete waiting equilibrium.

Proposition 2 A complete waiting equilibrium exists if and only if $\tau=1$ and $\Delta \leq \min \left\{\epsilon+\frac{1}{2}-\frac{\alpha}{4}, \frac{3}{4}(1-\alpha)\right\}$. In a complete waiting equilibrium $k_{2}=\frac{1}{2}+\Delta$.

Proof: Using the fact that no player invests in the first period,

$$
h\left(s_{2}^{i}, k\right)=s_{2}^{i}+E\left(n_{2} \mid s_{2}^{i}, k\right)-1-\Delta .
$$

Observe that $n_{2}\left(s_{2}^{i}, \theta\right)$ is weakly increasing in $\theta$, because as $\theta$ increases, (weakly) more players receive a signal $s_{2}^{i} \geq k_{2}$. Hence,

$$
E\left(n_{2} \mid s_{2}^{i}, k\right)=\int_{s_{2}^{i}-\epsilon}^{s_{2}^{i}+\epsilon} n_{2}(\theta, k) d \theta
$$

is weakly increasing in $s_{2}^{i}$. This implies that $h\left(s_{2}^{i}, k\right)$ is strictly increasing in $s_{2}^{i}$. Rewriting condition (5) gives

$$
h\left(s_{2}^{i}, k\right)=k_{2}+E\left(n_{2} \mid s_{2}^{i}=k_{2}, k\right)-1-\Delta=0 .
$$


It is easy to check that $E\left(n_{2} \mid s_{2}^{i}=k_{2}, k\right)=\frac{1}{2}$, and hence $k_{2}=\frac{1}{2}+\Delta$. Since $h(\cdot)$ is increasing, it is optimal for all players in the second period to invest if and only if $s_{2}^{i} \geq k_{2}$. We are left to check under what conditions it is optimal to refrain from investing in the first period, i.e. $g\left(s_{1}^{i}, k\right) \leq 0 \forall s_{1}^{i}$.

Consider a player with a signal $s_{1}^{i}>k_{2}+3 \epsilon$. This player knows in equilibrium that all (other) players invest in the second period. Hence,

$$
g\left(s_{1}^{i}, k\right)=s_{1}^{i}+\alpha-1-\tau\left[s_{1}^{i}-\Delta\right]
$$

Rewriting, yields $g\left(s_{1}^{i}, k\right)=(1-\tau) s_{1}^{i}-(1-\alpha)+\tau \Delta$. Fix any values of $\alpha, \Delta, \tau$. If $\tau<1$, there exists a sufficiently high $s_{1}^{i}$ such that $g\left(s_{1}^{i}, k\right)>0$, ruling out the existence of a complete waiting equilibrium in this case. Hence, a necessary condition for a complete waiting equilibrium is that $\tau=1$.

From now on, let $\tau=1$. If $s_{1}^{i} \leq k_{2}-\epsilon$, it is optimal for player $i$ not to invest in the second period, independent of whether he gets good or bad news. Thus, in this case

$$
g\left(s_{1}^{i}, k\right)=s_{1}^{i}+\alpha E\left(n_{2} \mid s_{1}^{i}, k\right)-1 .
$$

Since, by the same reasoning as above, $E\left(n_{2} \mid s_{1}^{i}, k\right)$ is weakly increasing, $g\left(s_{1}^{i}, k\right)$ is strictly increasing in this case and hence $g\left(s_{1}^{i}, k\right)<0 \forall s_{1}^{i}<k_{2}-\epsilon$ if and only if $g\left(s_{1}^{i}=\frac{1}{2}+\Delta-\epsilon, k\right) \leq 0$. Using Lemma (3), $E\left(n_{2} \mid s_{2}^{i}=k_{2}-2 \epsilon, k\right)=0$, and $E\left(n_{2} \mid s_{2}^{i}=k_{2}, k\right)=\frac{1}{2}$, this simplifies to

$$
\frac{1}{2}+\Delta-\epsilon+\frac{\alpha}{4}-1 \leq 0
$$

which can be rewritten as $\Delta \leq \epsilon+\frac{1}{2}-\frac{\alpha}{4}$. We conclude that $g\left(s_{1}^{i}, k\right)<0 \forall s_{1}^{i}<k_{2}-\epsilon$ if and only if $\Delta \leq \epsilon+\frac{1}{2}-\frac{a}{4}$.

If $s_{1}^{i} \in\left(k_{2}-\epsilon, k_{2}+\epsilon\right)$, then it is optimal to invest in the second period if and only if player $i$ receives good news. Thus, in this case

$$
\begin{gathered}
g\left(s_{1}^{i}, k\right)=s_{1}^{i}+\alpha E\left(n_{2} \mid s_{1}^{i}, k\right)-1-\frac{1}{2}\left(s_{1}^{i}+\epsilon\right)-\frac{1}{2} E\left(n_{2} \mid s_{2}^{i}=s_{1}^{i}+\epsilon, k\right)+\frac{\Delta}{2}+\frac{1}{2} \\
=\frac{1}{2}\left(s_{1}^{i}+\Delta-\epsilon\right)-\frac{1-\alpha}{2} E\left(n_{2} \mid s_{2}^{i}=s_{1}^{i}+\epsilon, k\right)+\frac{\alpha}{2} E\left(n_{2} \mid s_{2}^{i}=s_{1}^{i}-\epsilon, k\right)-\frac{1}{2} .
\end{gathered}
$$

Using Leibnitz's rule,

$\frac{\partial g\left(s_{1}^{i}, k\right)}{\partial s_{1}^{i}}=\frac{1}{2}-\frac{1-\alpha}{2}\left(n_{2}\left(\theta=s_{1}^{i}+2 \epsilon, k\right)-n_{2}\left(s_{1}^{i}, k\right)\right)+\frac{\alpha}{2}\left(n_{2}\left(s_{1}^{i}, k\right)-n_{2}\left(s_{1}^{i}-2 \epsilon, k\right)\right)$ 


$$
\geq \frac{1}{2}-\frac{1-\alpha}{2} \geq 0
$$

where the first inequality follows from the facts that $n_{2}(\theta, k)$ is weakly increasing and lies between 0 and 1 , and the second inequality follows from $\alpha \leq 1$. Hence $g\left(s_{1}^{i}, k\right) \leq 0 \forall s_{1}^{i} \in\left(k_{2}-\epsilon, k_{2}+\epsilon\right)$ if and only if $g\left(s_{1}^{i}=k_{2}+\epsilon, k\right) \leq 0$. Since $g\left(s_{1}^{i}=k_{2}+\epsilon, k\right)=\Delta-\frac{3}{4}(1-\alpha)$, we conclude that $g\left(s_{1}^{i}, k\right) \leq 0 \forall s_{1}^{i} \in\left(k_{2}-\epsilon, k_{2}+\epsilon\right)$ if and only if $\Delta \leq \frac{3}{4}(1-\alpha)$.

If $s_{1}^{i} \geq k_{2}+\epsilon$, then it is optimal for player $i$ to invest in the second period independent of whether he receives good news or bad news. Therefore, in this case $g\left(s_{1}^{i}, k\right)=\Delta-(1-\alpha) E\left(n_{2} \mid s_{1}^{i}, k\right)$. Hence $g\left(s_{1}^{i}, k\right)$ is weakly decreasing in $s_{1}^{i}$ and it again suffices to analyze whether $g\left(s_{1}^{i}=k_{2}+\epsilon, k\right) \leq 0$, which is the case if and only if $\Delta \leq \frac{3}{4}(1-\alpha)$. Q.E.D.

A complete waiting equilibrium exists only if the cost of waiting are not to high. If $\tau<1$ players discount the payoffs of investing in the second period as their benefits from investing are delayed. In this case, as the fundamental $\theta$ increases without bound, the forgone first-period benefit grows without bound. In other words, if $\tau<1$, then it is a dominant strategy to invest in the first period for sufficiently high signals. Thus a complete waiting equilibrium cannot exist in this case. If $\tau=1$ then the cost of delay are measured by $\Delta$. If $\Delta$ is to large, it is intuitive that no complete waiting equilibrium exists. Indeed, as $\Delta$ increases two complications may arise. First, players with a signal $s_{1}^{i}<1 / 2+\Delta-\epsilon$, who are meant to abstain from investing in an immediate investment equilibrium, may prefer to invest in the first period rather than not investing at all. For a given $\Delta$ this problem is more likely to arise if $\epsilon$ decreases, because in that case some of these players expect the state of the world to be better. Also this problem is more likely to arise as the inter-period network effect $\alpha$ increases and these players expect to benefit more from the second period investment activity. Second, players may prefer to invest immediately rather than to wait and invest in the second period. This problem is more likely to occur as the inter-period network effect $\alpha$ increase and early movers benefit more from the late investment activity. Depending on the parameters, either problem may arise first.

If there is no early mover cohort effect $(\alpha=1)$, then a complete waiting equilibrium cannot exist (unless there are no waiting costs at all, i.e. $\tau=1$ and $\Delta=0$ ). The reason is that in this case a player with a very high first period signal foresees that all players will invest. But if there are no cohort effects, he prefers to 
invest immediately and save the waiting cost. If there are cohort effects, however, he prefers to wait if the waiting cost are less than the reduction in the network effect from moving early. It is easy to check that if cohort effects are sufficiently strong, then there exist parameter values for which an immediate investment and a complete waiting equilibrium coexist. In the absence of cohort effects, this cannot be the case (except if there are no waiting costs at all). This result already hints at the main message of our paper: Cohort effects can give rise to multiple equilibria. The following analysis shows that this insight generalizes to the case where it is a dominant strategy for players with a very high first period signal to invest immediately (i.e. $\tau<1$ ).

We will refer to an equilibrium in which players with high signals invest immediately and player with intermediate signals wait and invest later when receiving good news (but not when receiving bad news) as an informative waiting equilibrium. Formally, an informative waiting equilibrium is an equilibrium in which $k_{1}-\epsilon<k_{2}<k_{1}+\epsilon$.

For brevity, let $x \equiv 4 \epsilon+\gamma$ and let

$$
\begin{gathered}
D \equiv-16 \Delta+16 \epsilon-8+12 \gamma+[(2-\alpha)-(2-\tau) x]^{2}, \\
\Delta^{a} \equiv-\frac{1}{2}+\frac{3}{4} \gamma+\epsilon+\frac{1}{16}[(2-\alpha)-(2-\tau) x]^{2}, \\
\Delta^{b} \equiv-\frac{1}{2}+\frac{3}{4} \gamma+\epsilon, \\
\Delta^{c} \equiv \frac{\gamma}{4}(1+\tau)-\frac{(1+\alpha)+4 \epsilon(1-\tau)}{4} .
\end{gathered}
$$

We are ready to characterize when an informative waiting equilibrium exists.

Proposition 3 There exists an informative waiting equilibrium $\left(k_{11}, k_{21}\right)$ if the following three conditions are satisfied: (a) $\Delta \leq \Delta^{a}$, (b) either $(2-\alpha)>(2-\tau) x$ or $\Delta \leq \Delta^{b}$, and (c) $\Delta>\Delta^{c}$. In this informative waiting equilibrium

$$
\begin{gathered}
k_{11}=\frac{1}{8}\left\{\tau(\tau-2) x^{2}+2 x[1-(1-\alpha)(1-\tau)]+(1-\alpha)^{2}+3+(x \tau-\alpha) \sqrt{D}\right\}, \\
k_{21}=\frac{1}{8}\left\{-\sqrt{D}(\alpha+8 \epsilon-x \tau)+x^{2} \tau^{2}+2 \tau x(1-\alpha-4 \epsilon-x)+(2-\alpha)^{2}-8(1-\alpha) \epsilon+2 \alpha(1+x)+16 \epsilon x\right\} .
\end{gathered}
$$


Furthermore, there exists an informative waiting equilibrium $\left(k_{12}, k_{22}\right)$ if the following three conditions are satisfied: (a) $\Delta \leq \Delta^{a}$, (d) $(2-\alpha)>(2-\tau) x$, and (e) $\Delta>\Delta^{b}$. In this informative waiting equilibrium

$$
\begin{gathered}
k_{12}=\frac{1}{8}\left\{\tau(\tau-2) x^{2}+2 x[1-(1-\alpha)(1-\tau)]+(1-\alpha)^{2}+3-(x \tau-\alpha) \sqrt{D}\right\}, \\
k_{22}=\frac{1}{8}\left\{\sqrt{D}(\alpha+8 \epsilon-x \tau)+x^{2} \tau^{2}+2 \tau x(1-\alpha-4 \epsilon-x)+(2-\alpha)^{2}-8(1-\alpha) \epsilon+2 \alpha(1+x)+16 \epsilon x\right\} .
\end{gathered}
$$

Conversely, there exists no other informative waiting equilibrium.

Proof: Rewriting (4) and (5) using the fact that $k_{1}-\epsilon<k_{2}<k_{1}+\epsilon$ in an informative waiting equilibrium gives

$$
\begin{gathered}
k_{1}+\frac{1}{2}+\left(\frac{\alpha}{8 \epsilon}\right)\left(k_{1}+\epsilon-k_{2}\right)-1-\frac{\tau}{2}\left\{k_{1}+\epsilon+\frac{3 \gamma}{4}+\frac{1}{16 \epsilon^{2}}\left(k_{1}+\epsilon-k_{2}\right)\left(k_{2}+3 \epsilon-k_{1}\right)-1-\Delta\right\}=0, \\
k_{2}+\gamma\left\{\frac{1}{4}+\frac{1}{4 \epsilon}\left(k_{2}+\epsilon-k_{1}\right)+\frac{1}{16 \epsilon^{2}}\left(k_{1}+\epsilon-k_{2}\right)\left(k_{2}+3 \epsilon-k_{1}\right)\right\}-1-\Delta=0 .
\end{gathered}
$$

Thus, (4) and (5) are a pair of quadratic equations, which is equivalent to a fourth order polynomial. Hence, there exists a routine procedure to solve this system of equations. Using mathematica to solve this system of equations shows that there are only two pair of roots $\left(k_{11}, k_{21}\right)$ and $\left(k_{21}, k_{22}\right)$. Rewriting, gives the expressions given in the proposition above. Because (4) and (5) are necessary conditions for an equilibrium, all informative waiting equilibria ar either of the form $\left(k_{11}, k_{21}\right)$ or $\left(k_{21}, k_{22}\right)$.

Observe that all roots are real if and only if $D \geq 0$. This requires that

$$
16 \epsilon-8+12 \gamma+[(2-\alpha)-(2-\tau) x]^{2} \geq 16 \Delta .
$$

Rewriting gives condition (a).

$\left(k_{11}, k_{21}\right)$ is a valid solution only if $k_{11}-\epsilon<k_{21}<k_{11}+\epsilon$, because otherwise the functional form of (4) and (5) would differ from the one used above. That is, we require that (i) $-\epsilon<k_{11}-k_{21}$ and that (ii) $k_{11}-k_{21}<\epsilon$. Using the fact that

$$
k_{11}-k_{21}=\epsilon[1-\alpha-(2-\tau) x+\sqrt{D}],
$$

condition (i) holds if and only if

$$
(2-\tau) x-(2-\alpha)<\sqrt{D} .
$$


Note that this inequality is satisfied if either $(2-\alpha)>(2-\tau) x$ or if

$$
[(2-\tau) x-(2-\alpha)]^{2}<-16 \Delta+16 \epsilon-8+12 \gamma+[(2-\alpha)-(2-\tau) x]^{2} .
$$

Rewriting gives condition (b).

Using $k_{11}-k_{21}=\epsilon[1-\alpha-(2-\tau) x+\sqrt{D}]$, to rewrite condition (ii) gives

$$
\sqrt{D}<\alpha+(2-\tau) x
$$

Squaring this inequality on both sides and rewriting yields

$$
-16 \Delta+16 \epsilon+12 \gamma-4(1+\alpha)-4 x(2-\tau)<0,
$$

which is equivalent to condition (c) in the proposition. Hence, conditions (a), (b), and $(\mathrm{c})$ are necessary conditions for $\left(k_{11}, k_{21}\right)$ to characterize an equilibrium.

Similar, $\left(k_{12}, k_{22}\right)$ is a valid solution only if both (i) $-\epsilon<k_{12}-k_{22}$ and (ii) $k_{12}-k_{22}<$ $\epsilon$ hold. Using the fact that $k_{12}-k_{22}=\epsilon[1-\alpha-(2-\tau) x-\sqrt{D}]$, condition (i) holds if and only if $\sqrt{D}<(2-\alpha)-(2-\tau) x$. Hence, condition (i) requires that $(2-\alpha)>(2-\tau) x$, which is condition $(\mathrm{d})$ in the proposition, and that $D<[(2-\alpha)-(2-\tau) x]^{2}$, which is equivalent to condition (e) in the proposition. We conclude that conditions (a), (d) and (e) are necessary conditions for $\left(k_{12}, k_{22}\right)$ to characterize an equilibrium. (Note also that $k_{12}-k_{22}=\epsilon[1-\alpha-(2-\tau) x-\sqrt{D}]<\epsilon$.) Hence, we have established that no other informative waiting equilibrium than the ones characterized in the proposition exist. To show that $\left(k_{11}, k_{21}\right)$ and $\left(k_{12}, k_{22}\right)$ are indeed equilibria under the above conditions, we are left to verify that (i) $h\left(s_{2}^{i}, k\right)<0$ for all $s_{2}^{i}<k_{2}$, (ii) $h\left(s_{2}^{i}, k\right)>0 \forall s_{2}^{i} \in\left(k_{2}, k_{1}+\epsilon\right]$, and that (iii) $g\left(s_{1}^{i}, k\right)<0$ if and only if $s_{1}^{i}<k_{1}$. Conditions (i) and (ii) follow from Lemma (4) in Appendix 2, and condition (iii) follows from Lemmas (7),(9), and (11) to (14). Q.E.D.

To understand under what conditions an informative investment equilibrium exists, suppose first that $(2-\alpha)<(2-\tau) x$, as is the case in the absence of cohort effects. Then, since condition $(\mathrm{d})$ is violated, the $\left(k_{12}, k_{22}\right)$ equilibrium does not exist. Next, observe that in this case conditions (a) and (b) are satisfied whenever $\Delta$ is to low to sustain an immediate investment equilibrium, i.e. when waiting to act on more information is profitable. The role of condition (c) is to ensure that the relevant decision for a player with signal $s_{1}^{i}=k_{1}$ is whether to wait for good news or whether to invest immediately. If it is violated, the player would prefer 
to invest in the second period also when getting bad news (which explains why condition (c) gives a lower bound on $\Delta$ ). Thus condition (c) can only be binding in the presence of cohort effects. If cohort effects are absent, the only reason to wait is to collect information in order to make a better informed decision. So if a player would prefer to invest when getting bad news, he could invest immediately and save the waiting costs. If cohort effects are present, however, one may want to wait in order to benefit from a higher network effect. Note that this requires that $\gamma$ is sufficiently greater than $\alpha$; that is late movers must enjoy a higher intra-period network effect than early movers. The intuition for this is that a player with signal $s_{1}^{i}=k_{1}$ expects half of the population to invest in the first period. So he can expect at most half of the population to invest late. Therefore, he can only expect to gain a larger network effect by moving late if the inter-period network effect for late movers $\gamma$ is greater than its first period counterpart $\alpha$.

We are left to consider the case in which $(2-\alpha)>(2-\tau) x$. Trivially, this implies that conditions (b) and (d) are satisfied. Clearly, then the equilibrium $\left(k_{11}, k_{21}\right)$ exists for all $\Delta \in\left(\Delta^{c}, \Delta^{a}\right]$ and the equilibrium $\left(k_{21}, k_{22}\right)$ exists for all $\Delta$ in the nonempty interval $\left(\Delta^{b}, \Delta^{a}\right]$. Since an immediate investment equilibrium exists for all $\Delta>\Delta^{b}$, this implies that if $(2-\alpha)>(2-\tau) x$, there exist values of $\Delta$ for which our model has multiple equilibria as long as $\Delta^{a} \geq 0$. To better understand when $(2-\alpha)>(2-\tau) x$, it is useful to rewrite this condition as $(1-\alpha)+(1-\gamma)+(\tau \gamma-\gamma)>(2-\tau) 4 \epsilon$. First, observe that this condition cannot be satisfied in the absence of cohort effects (i.e $\alpha=\gamma=1$ ). But as either cohort effects increases (i.e. $\alpha$ or $\gamma$ decreases), the condition is more likely to be satisfied. One interpretation of this fact is that as cohort become more important, dynamic coordination becomes more important. A player then only wants to invest if he believes that the other players invest at the same point in time. Second, if $\tau<1$, then decreasing $\gamma$ is more likely to make this condition hold then decreasing $\alpha$ by the same amount, reflecting the fact that a first-period decision maker discounts the second-period investment payoffs. Third, as the uncertainty $\epsilon$ increases, the conditions is less likely to hold. As $\epsilon$ increases the uncertainty about the fundamental becomes more important relative to the coordination aspect. As the coordination aspect becomes less important, multiple equilibria are less likely to exists.

The following Lemma rules out the existence of other symmetric switching equilibria in various of the economic environments discussed in Section 3. 
LEMma 1 If $\frac{1}{2}(\tau \gamma-\alpha)-\epsilon(1-\tau)<\Delta$ then there exists no equilibrium in the class of symmetric switching equilibria in which $k_{2}<k_{1}-\epsilon<\infty$.

Proof: Recall that $E\left(n_{1} \mid s_{1}^{i}=k_{1}, k\right)=\frac{1}{2}$. Also, note that since $k_{2}<k_{1}-\epsilon$, a player with signal $s_{1}^{i}=k_{1}$ who waits will invest in the second period for certain. Using these facts and Lemma (3) to rewrite equilibrium conditions (4) and (5) gives:

$$
\begin{gathered}
k_{1}(1-\tau)+\frac{1}{2}(1-\tau \gamma)+(\alpha-\tau) E\left(n_{2} \mid s_{1}^{i}=k_{1}, k\right)-1+\tau(1+\Delta)=0, \\
k_{2}=1+\Delta-\gamma E\left(n_{1} \mid s_{2}^{i}=k_{2}, k\right)-E\left(n_{1} \mid s_{2}^{i}=k_{2}, k\right) .
\end{gathered}
$$

Observe that any player with a signal $s_{2}^{i}<k_{2}$ does not invest in either period because in this case $s_{1}^{i} \leq s_{2}^{i}+\epsilon<k_{2}+\epsilon<k_{1}$. Hence, $E\left(n_{1}+n_{2} \mid s_{2}^{i}=k_{2}, k\right)=\frac{1}{2}$ and thus $E\left(\gamma n_{1}+n_{2} \mid s_{2}^{i}=k_{2}, k_{2}\right) \leq \frac{1}{2}$. Using this fact and equation (8), we conclude that $k_{2} \geq \frac{1}{2}+\Delta$.

Rewriting equation (7) shows that

$$
k_{1}=\frac{1}{1-\tau}\left\{\frac{1}{2}\left(1+\tau \gamma-\tau(1+\Delta)+(\tau-\alpha) E\left(n_{2} \mid s_{1}^{i}=k_{1}, k\right)\right)\right\} .
$$

First, suppose that $\alpha \geq \tau$. In this case

$$
k_{1} \leq \frac{1}{1-\tau}\left\{\frac{1}{2}(1+\tau \gamma-\tau(1+\Delta)\}\right.
$$

and since $k_{2} \geq \frac{1}{2}+\Delta$ a necessary condition for $k_{1}>k_{2}+\epsilon$ is that

$$
\left\{\frac{1}{2}(1+\tau \gamma)-\tau(1+\Delta)\right\} \geq(1-\tau)\left(\frac{1}{2}+\Delta+\epsilon\right) .
$$

This is equivalent to $0 \geq \Delta+\epsilon(1-\tau)+\frac{\tau}{2}(1-\gamma)$, a contradiction.

We are left to consider the case in which $\alpha<\tau$. Observe that since $E\left(n_{1} \mid s_{1}^{i}=\right.$ $\left.k_{1}, k\right)=\frac{1}{2}$ and $n_{1}+n_{2} \leq 1, E\left(n_{2} \mid s_{1}^{i}=k_{1}, k\right) \leq \frac{1}{2}$. Hence,

$$
k_{1} \leq \frac{1}{1-\tau}\left\{\frac{1}{2}\left(1+\tau \gamma-\tau(1+\Delta)+(\tau-\alpha) \frac{1}{2}\right\} .\right.
$$

Thus, a necessary condition for $k_{1}>k_{2}+\epsilon$ is that

$$
\left\{\frac{1}{2}\left(1+\tau \gamma-\tau(1+\Delta)+(\tau-\alpha) \frac{1}{2}\right\} \geq(1-\tau)\left(\frac{1}{2}+\Delta+\epsilon\right) .\right.
$$


Rewriting this condition establishes the Lemma. Q.E.D.

The Lemma gives parameter restrictions that rule out a symmetric switching equilibrium in which players wait in order to invest in the second period with certainty. This type of behavior rules out any informational reason for waiting. Rather waiting must be driven by the desire to coordinate the timing of the investment. This can only be profitable if there are cohort effects and if, as discussed above, the second-period cohort effect is sufficiently less than the first-period cohort effect (i.e. $\gamma>\alpha$ ).

We are left to prove that there exists a unique equilibrium in the absence of cohort effects. We first observe that this is true within the class of symmetric switching strategies.

Proposition 4 Suppose there are no cohort effects (i.e. $\alpha=\gamma=1$ ) and positive waiting costs (i.e. either $\tau<1$ or $\Delta>0$ ). Then there exists a unique equilibrium in symmetric switching strategies.

Proof: Rewriting conditions (a), (b) and (c) from Proposition (3) yields:

$$
\begin{gathered}
\Delta \leq \frac{1}{4}+\epsilon+\frac{1}{16}(-1-8 \epsilon+4 \epsilon \tau+\tau)^{2}, \\
\Delta \leq \frac{1}{4}+\epsilon, \\
-(1-\tau)\left(\frac{1}{4}+\epsilon\right) \leq 0 \leq \Delta .
\end{gathered}
$$

Hence, there exists an informative waiting equilibrium $\left(k_{11}, k_{21}\right)$ if $0 \leq \Delta \leq \frac{1}{4}+\epsilon$. Note also that, when $\alpha=\gamma=1$, the other informative waiting equilibrium $\left(k_{12}, k_{22}\right)$ cannot exist because condition (d) can be rewritten as $1>(2-\tau)(1+4 \epsilon)$, which contradicts the fact that $\tau \leq 1$ and $\epsilon>0$. Finally, observe that, when $\alpha=\gamma=1$, the condition stated in Lemma (1) can be written as $-(1-\tau)\left(\frac{1}{2}+\epsilon\right)<\Delta$. This condition is always satisfied unless $\tau=1$ and $\Delta=0$. From Proposition (1), it follows that an immediate investment equilibrium exists if and only if $\Delta \geq \frac{1}{4}+\epsilon$. Furthermore, as $h()$ is strictly increasing for $\alpha=\gamma=1$, the immediate investment equilibrium is unique. Q.E.D.

In Appendix 3, we extend this result to show that there exists a unique equilibrium in rationalizable strategies in the absence of cohort effects. 
Proposition 5 Suppose there are no cohort effects (i.e. $\alpha=\gamma=1$ ) and positive waiting costs (i.e. either $\tau<1$ or $\Delta>0$ ). Then the symmetric switching equilibrium strategy is the (essentially) unique rationalizable equilibrium.

\section{Summary and Discussion}

In this paper, we analyzed the impact of strategic waiting in a global game. In contrast to its static counterpart, we found that the nature of the network effect is important. In particular, allowing for cohort effects can reintroduce multiple equilibria in environments in which the static game has a unique equilibrium. Furthermore, we argued that this finding is important because cohort effects arise naturally in many dynamic coordination problems.

Table 1 presents parameter conditions under which an immediate investment equilibrium, a complete waiting equilibrium, and an informative waiting equilibrium exist in various economic environments encompassed by our model. In the absence of cohort effects, our game has a unique equilibrium (neglecting the knife edge case in which their are no waiting costs and investors with a sufficiently high signal are indifferent between investing early and waiting in order to invest late). The PIEMA model is an interpretation of our model in which there are no cohort effects, and thus in the PIEMA environment there always exists a unique equilibrium.

Perhaps more surprisingly, in the FHTAP interpretation our model has a unique equilibrium in the class o symmetric switching strategies even though this interpretation allows for an early mover cohort effect. In this economic environment, however, the second period intra- and inter-period network effects are equal to each other. Furthermore, the first period intra-period network effect is simply the discounted second-period network effect. In this environment, therefore, the benefits of waiting and investing in the second period depend only on the total mass of investors and not on when the other players invest. Similarly, the benefits of investing in the second period depend only on the total mass of investors and not on the timing of the other players' investments. Intuitively, therefore, the dynamic coordination is not severe enough for multiple equilibria to arise.

The ATFL interpretation, in contrast, allows for both a first- and a second-period cohort effect. The dynamic coordination aspect thus becomes more important and 
our model has multiple equilibria in such an environment. This result carries over to the TAPPCT environment because it is very flexible and one can reproduce the parameter of the ATFL interpretation. In summary, the nature of the cohort effects determines whether our dynamic global game has multiple equilibria. These multiple equilibria did arise even though we used a Laplacian prior, abstracted from social learning, and restricted attention to the class of symmetric switching strategies.

\section{Appendix 1}

We first prove the following lemma:

LEMMA $2 \forall k_{2} \in\left(k_{1}-\epsilon, k_{1}+\epsilon\right)$, one has:

$$
n_{1}(\theta, k)= \begin{cases}0 & \text { if } \theta<k_{1}-2 \epsilon, \\ \frac{2 \epsilon+\theta-k_{1}}{4 \epsilon} & \text { if } k_{1}-2 \epsilon \leq \theta<k_{1}+2 \epsilon \\ 1 & \text { if } k_{1}+2 \epsilon \leq \theta\end{cases}
$$

and,

$$
n_{2}(\theta, k)= \begin{cases}0 & \text { if } \theta<k_{2}-\epsilon, \\ \frac{\epsilon-k_{2}+\theta}{4 \epsilon} & \text { if } k_{2}-\epsilon \leq \theta<k_{1}, \\ \frac{k_{1}+\epsilon-k_{2}}{4 \epsilon} & \text { if } k_{1} \leq \theta<k_{2}+\epsilon, \\ \frac{k_{1}+2 \epsilon-\theta}{4 \epsilon} & \text { if } k_{2}+\epsilon \leq \theta<k_{1}+2 \epsilon, \\ 0 & \text { if } k_{1}+2 \epsilon \leq \theta,\end{cases}
$$

Proof: To compute $n_{1}(\cdot)$ and $n_{2}(\cdot)$ we will work with the following graph (or with variants thereof):

\section{[Insert here Graph 1]}

In the above graph, the two "thick" black lines represent all the possible realizations that $\left(\epsilon_{2}^{i}, \epsilon_{1}^{i}\right)$ can take. For example, player $a$ in the graph received a $\epsilon_{1}^{i}=\epsilon_{2}^{i}=-\epsilon$, 
while player $d$ received $\epsilon_{1}^{i}=\epsilon_{2}^{i}=\epsilon$. All the players who received a $\epsilon_{1}^{i}=-\epsilon$ are situated on the lower thick line. Those players will receive "good news" at time two. Similarly, all players situated on the upper thick line will receive "bad news" at time two. Actually, one can best think of the graph above as possessing a third dimension representing $f\left(\left(s_{1}^{i}, s_{2}^{i}\right) \mid \theta\right)$. From above, we know that $\operatorname{Pr}\left(\epsilon_{1}^{i}=\epsilon\right)=\frac{1}{2}$, that $\epsilon_{2}^{i}$ is independent of $\epsilon_{1}^{i}$ and that $\epsilon_{2}^{i}$ is drawn from a uniform distribution. Hence, we know that half of our population receive an $\epsilon_{1}^{i}=-\epsilon$ and lie, uniformly distributed, on the lower thick black line, while the other half lie, uniformly distributed, on the upper thick black line. Therefore, this third dimension is "trivial" and is not shown in the graph.

The diagonal " $k_{1}-\theta$ " represents the combination of all $\left(\epsilon_{2}^{i}, \epsilon_{1}^{i}\right)$ such that $\epsilon_{1}^{i}+\epsilon_{2}^{i}=$ $k_{1}-\theta$. All players who lie to its right and above possess a first-period signal $s_{1}^{i}>k_{1}$, since $s_{1}^{i}=\theta+\epsilon_{1}^{i}+\epsilon_{2}^{i} \geq k_{1}$ if and only if $\epsilon_{1}^{i}+\epsilon_{2}^{i} \geq k_{1}-\theta$. Hence, the diagonal $k_{1}-\theta$ permits us to compute the mass of period one investors.

$e$ denotes the point in which the diagonal $k_{1}-\theta$ cuts the upper thick black line. What are the coordinates of point $e$ ? We know that all points on the diagonal satisfy the restriction that their $x$ and $y$ coordinates sum up to $k_{1}-\theta$. We also know that in point $e$ the $y$ coordinate equals $+\epsilon$. Therefore the coordinates of point $e$ are $\left(k_{1}-\theta-\epsilon, \epsilon\right)$. If $k_{1}=\theta$, then the diagonal goes through the points $b$ and $c$ (this is logical: in both points $\left.k_{1}-\theta=\theta-\theta=0=\epsilon-\epsilon\right)$. If $k_{1}=\theta-2 \epsilon$, then the diagonal goes through the point $a$. This is because in the point $a, k_{1}-\theta=-2 \epsilon=-\epsilon-\epsilon$. Similarly, if $k_{1}=\theta+2 \epsilon$, then the diagonal goes through the point $d$. By continuity, if $\theta-2 \epsilon<k_{1}<\theta$, the diagonal $k_{1}-\theta$ cuts the thick line situated on the $\mathrm{X}$-axis. Similarly, if $\theta<k_{1}<\theta+2 \epsilon$, the diagonal cuts the upper thick line.

The vertical " $k_{2}-\theta$ " permits us to compute the mass of players who invest at time two. For example, in graph three all players situated on the X-axis and to the right of " $k_{2}-\theta$ " invest at time two. This is easy to see: a player having received an $\epsilon_{1}^{i}=-\epsilon$ invests at time two if and only if $s_{2}^{i}=\theta+\epsilon_{2}^{i}>k_{2}$ or if and only if $\epsilon_{2}^{i}>k_{2}-\theta . f$ denotes the point in which the vertical $k_{2}-\theta$ cuts the $\mathrm{X}$-axis.

When doesn't the vertical $k_{2}-\theta$ cross the lower thick line? $k_{2}-\theta>\epsilon$ if and only if $k_{2}-\epsilon>\theta$. This is intuitive: if $\theta$ is "low", then no player who received "good news" will invest at time two. In that case point $f$ lies to the right of point $b$. Similarly, $k_{2}-\theta<-\epsilon$ if and only if $k_{2}+\epsilon<\theta$. In hat case point $f$ lies to the left 
of point $a$.

We are focusing on an equilibrium in which $k_{1}-\epsilon<k_{2}<k_{1}+\epsilon$. This implies that:

$$
k_{1}-2 \epsilon<k_{2}-\epsilon<k_{1}<k_{2}+\epsilon<k_{1}+2 \epsilon .
$$

Therefore we must consider the following six cases:

(i) $\theta<k_{1}-2 \epsilon$,

(ii) $k_{1}-2 \epsilon<\theta<k_{2}-\epsilon$,

(iii) $k_{2}-\epsilon<\theta<k_{1}$,

(iv) $k_{1}<\theta<k_{2}+\epsilon$,

(v) $k_{2}+\epsilon<\theta<k_{1}+2 \epsilon$,

(vi) $k_{1}+2 \epsilon<\theta$.

In case (i) we know that $\theta<k_{1}-2 \epsilon<k_{2}-\epsilon$. From above, we know that this implies that points $e$ and $f$ lie to the right of (respectively) $d$ and $b$. Hence, $n_{1}\left(\theta<k_{1}-2 \epsilon, k\right)=n_{2}\left(\theta<k_{1}-2 \epsilon, k\right)=0$.

In case (ii) we know that point $f$ lies to the right of point $b$, implying that - due to a low $\theta-n_{2}\left(k_{1} 2 \epsilon<\theta<k_{2} \epsilon, k\right)=0$. Moreover we also know that in this case $\theta<k_{1}$ which implies that the diagonal $k_{1}-\theta$ cuts the upper thick line. This case is represented in graph 2 .

\section{[Insert here Graph 2]}

In this case all players situated between points $e$ and $d$ invest at time one. Hence, it is straightforward to compute that $n_{1}\left(k_{1}-2 \epsilon<\theta<k_{2}-\epsilon, k\right)=\frac{2 \epsilon+\theta-k_{1}}{4 \epsilon}$.

In case (iii) $\theta$ is still strictly lower than $k_{1}$ but the vertical $k_{2}-\theta$ crosses the two thick black lines. This case is represented in graph 1 . The coordinates of $e$ are $\left(k_{1}-\theta-\epsilon, \epsilon\right)$ and the ones of point $f^{\prime}$ are $\left(k_{2}-\theta, \epsilon\right)$. We are focussing on an equilibrium in which $k_{2}>k_{1}-\epsilon$. This last inequality can be rewritten as $k_{2}-\theta>k_{1}-\theta-\epsilon$ which amounts to stating that point $f^{\prime}$ always lies to the right of point $e$. From above we thus know that $n_{1}\left(k_{2}-\epsilon<\theta<k_{1}, k\right)=\frac{2 \epsilon+\theta-k_{1}}{4 \epsilon}$. All players lying between $[f, b]$ invest at time two. Hence, $n_{2}\left(k_{2}-\epsilon<\theta<k_{1}, k\right)=\frac{\epsilon-k_{2}+\theta}{4 \epsilon}$.

In case (iv), $\theta$ is higher than $k_{1}$. This implies that the diagonal " $k_{1}-\theta$ " cuts the lower thick line. Therefore all players who received an $\epsilon_{1}^{i}=\epsilon$ (and who are thus 
situated on the upper thick line) invest at time one. This case is represented in graph 3 .

\section{[Insert here Graph 3]}

From above we know that the coordinates of point $e$ are $\left(k_{1}-\theta+\epsilon,-\epsilon\right)$. The coordinates of point $f$ are $\left(k_{2}-\theta,-\epsilon\right)$. Note that $k_{2}-\theta<k_{1}-\theta+\epsilon$ if and only if $k_{2}<k_{1}+\epsilon$. As we work here under the assumption that $k_{2}<k_{1}+\epsilon$, it follows that point $f$ lies to the left of point $e$. From the graph it should be clear that $n_{1}\left(k_{1}<\theta<k_{2}+\epsilon, k\right)=\frac{1}{2}+\frac{\epsilon-k_{1}+\theta-\epsilon}{4 \epsilon}=\frac{2 \epsilon+\theta-k_{1}}{4 \epsilon}$ and that $n_{2}\left(k_{1}<\theta<k_{2}+\epsilon, k\right)$ $=\frac{k_{1}-\theta+\epsilon}{4 \epsilon}-\frac{k_{2}-\theta}{4 \epsilon}=\frac{k_{1}+\epsilon-k_{2}}{4 \epsilon}$.

In case (v) point $f$ lies to the left of point $a$. From above it should be clear that $n_{1}\left(k_{2}+\epsilon<\theta<k_{1}+2 \epsilon, k\right)=\frac{2 \epsilon+\theta-k_{1}}{4 \epsilon}$, and that $n_{2}\left(k_{2}+\epsilon<\theta<k_{1}+2 \epsilon, k\right)=\frac{k_{1}+2 \epsilon-\theta}{4 \epsilon}$.

In case (vi) point $e$ (see graph 3) lies to the left of point $a$. Therefore $n_{1}\left(k_{1}+2 \epsilon<\right.$ $\theta, k)=1$ and $n_{2}\left(k_{1}+2 \epsilon<\theta, k\right)=0$. Q.E.D.

\section{$7 \quad$ Appendix 2}

LEMma $3 E\left(n_{j} \mid s_{1}^{i}, k\right)=\frac{1}{2} E\left(n_{j} \mid s_{2}^{i}=s_{1}^{i}+\epsilon, k\right)+\frac{1}{2} E\left(n_{j} \mid s_{2}^{i}=s_{1}^{i}-\epsilon, k\right) \forall j=1,2$.

Proof: Trivially, one has

$$
\begin{gathered}
E\left(n_{j} \mid s_{1}^{i}, k\right)=\frac{1}{4 \epsilon} \int_{s_{1}^{i}-2 \epsilon}^{s_{1}^{i}+2 \epsilon} n_{j}(\theta, k) d \theta \\
=\frac{1}{2}\left\{\frac{1}{2 \epsilon} \int_{s_{1}^{i}-2 \epsilon}^{s_{1}^{i}} n_{j}(\theta, k) d \theta+\frac{1}{2 \epsilon} \int_{s_{1}^{i}}^{s_{1}^{i}+2 \epsilon} n_{j}(\theta, k) d \theta\right\} \\
=\frac{1}{2} E\left(n_{j} \mid s_{2}^{i}=s_{1}^{i}+\epsilon, k\right)+\frac{1}{2} E\left(n_{j} \mid s_{2}^{i}=s_{1}^{i}-\epsilon, k\right) .
\end{gathered}
$$

Q.E.D.

Lemma 4 For any $k$ that solves equations (4) and (5) and for which $k_{2} \in\left(k_{1}-\right.$ $\left.\epsilon, k_{1}+\epsilon\right)$, one has $h\left(s_{2}^{i}, k\right)<0$ if $s_{2}^{i}<k_{2}$ and $h\left(s_{2}^{i}, k\right)>0$ if $s_{2}^{i} \in\left(k_{2}, k_{1}+\epsilon\right)$. 
Proof: Since

$$
h\left(s_{2}^{i}, k\right)=s_{2}^{i}+\int_{s_{2}^{i}-\epsilon}^{s_{2}^{i}+\epsilon}\left[\gamma n_{1}(\theta, k)+n_{2}(\theta, k)\right] d \theta-1-\Delta
$$

Leibnitz's rule implies that

$$
\frac{\partial h\left(s_{2}^{i}, k\right)}{\partial s_{2}^{i}}=1+\left[\gamma n_{1}\left(s_{2}^{i}+\epsilon, k\right)+n_{2}\left(s_{2}^{i}+\epsilon, k\right)-\left(\gamma n_{1}\left(s_{2}^{i}-\epsilon, k\right)+n_{2}\left(s_{2}^{i}-\epsilon, k\right)\right)\right] .
$$

We have shown in Appendix 1 that $n_{1}(\cdot)$ is weakly increasing in $\theta$ and therefore a sufficient condition for $h(\cdot)$ to be strictly increasing is that

$$
n_{2}\left(s_{2}^{i}+\epsilon, k\right) \geq n_{2}\left(s_{2}^{i}-\epsilon, k\right) .
$$

By Appendix 1, $n_{2}(\cdot)$ is weakly increasing in $\theta$ for all $\theta \leq k_{2}+\epsilon$ and hence $h\left(s_{2}^{i}, k\right)$ is a strictly increasing function in $s_{2}^{i}$ for all $s_{2}^{i} \leq k_{2}$. Since $k$ solves the equations (4) and (5), $h\left(k_{2}, k\right)=0$ and we conclude that $h\left(s_{2}^{i}, k\right)<0$ if $s_{2}^{i}<k_{2}$.

Next, consider $s_{2}^{i} \in\left(k-2, k_{1}+\epsilon\right)$. Since $h\left(k_{2}, k\right)=0$, one can rewrite $h\left(s_{2}^{i}, k\right)$ as

$h\left(s_{2}^{i}, k\right)=\left(s_{2}^{i}-k_{2}\right)+\gamma\left[E\left(n_{1} \mid s_{2}^{i}, k\right)-E\left(n_{1} \mid s_{2}^{i}=k_{2}, k\right)\right]+\left[E\left(n_{2} \mid s_{2}^{i}, k\right)-E\left(n_{2} \mid s_{2}^{i}=k_{2}, k\right)\right]$.

As $s_{2}^{i}>k_{2}$, the first term is positive. Since, by Appendix $1, n_{1}(\theta, k)$ is weakly increasing in $\theta$, Leibnitz's rule implies that $E\left(n_{1} \mid s_{2}^{i}, k\right)$ is weakly increasing in $s_{2}^{i}$. Hence $\left[E\left(n_{1} \mid s_{2}^{i}, k\right)-E\left(n_{1} \mid s_{2}^{i}=k_{2}, k\right)\right] \geq 0$. Thus a sufficient condition for $h\left(s_{2}^{i}, k\right)>0$ is that

$$
\left[E\left(n_{2} \mid s_{2}^{i}, k\right)-E\left(n_{2} \mid s_{2}^{i}=k_{2}, k\right)\right] \geq 0
$$

To prove that condition (9) is satisfied, we establish below that (i) $E\left(n_{2} \mid s_{2}^{i}, k\right)$ is a concave function in $s_{2}^{i}$ for all $s_{2}^{i} \in\left(k_{2}, k_{1}+\epsilon\right)$, and that (ii) $E\left(n_{2} \mid s_{2}^{i}=k_{1}+\epsilon, k\right)=$ $E\left(n_{2} \mid s_{2}^{i}=k_{2}, k\right)$. By Leibnitz's rule,

$$
\frac{\partial E\left(n_{2} \mid s_{2}^{i}, k\right)}{\partial s_{2}^{i}}=\frac{1}{2 \epsilon}\left[n_{2}\left(s_{2}^{i}+\epsilon, k\right)-n_{2}\left(s_{2}^{i}-\epsilon, k\right)\right]
$$

and thus

$$
\frac{\partial^{2} E\left(n_{2} \mid s_{2}^{i}, k\right)}{\partial\left(s_{2}^{i}\right)^{2}}=\frac{1}{2 \epsilon}\left[\frac{\partial n_{2}\left(s_{2}^{i}+\epsilon, k\right)}{\partial s_{2}^{i}}-\frac{\partial n_{2}\left(s_{2}^{i}-\epsilon, k\right)}{\partial s_{2}^{i}}\right] .
$$

Using the facts that $k_{2}+\epsilon<s_{2}^{i}+\epsilon<k_{1}+2 \epsilon, k_{2}-\epsilon<s_{2}^{i}-\epsilon<k_{1}$ and Appendix 1 , it is easy to check that $\frac{\partial^{2} E\left(n_{1} \mid s_{2}^{i}, k\right)}{\partial\left(s_{2}^{i}\right)^{2}}=-\frac{1}{4 \epsilon^{2}}$. 
We are left to show that $E\left(n_{2} \mid s_{2}^{i}=k_{1}+\epsilon, k\right)=E\left(n_{2} \mid s_{2}^{i}=k_{2}, k\right)$. Using Appendix 1, one has

$$
E\left(n_{2} \mid s_{2}^{i}=k_{1}+\epsilon, k\right)=\frac{1}{2 \epsilon} \int_{k_{1}}^{k_{2}+\epsilon} \frac{k_{1}+\epsilon-k_{2}}{4 \epsilon} d \theta+\frac{1}{2 \epsilon} \int_{k_{2}+\epsilon}^{k_{1}+2 \epsilon} \frac{2 \epsilon+k_{1}-\theta}{4 \epsilon} d \theta,
$$

and

$$
E\left(n_{2} \mid s_{2}^{i}=k_{2}, k\right)=\frac{1}{2 \epsilon} \int_{k_{2}-\epsilon}^{k_{1}} \frac{\epsilon-k_{2}+\theta}{4 \epsilon} d \theta+\frac{1}{2 \epsilon} \int_{k_{1}}^{k_{2}+\epsilon} \frac{k_{1}+\epsilon-k_{2}}{4 \epsilon} d \theta
$$

Thus

$E\left(n_{2} \mid s_{2}^{i}=k_{1}+\epsilon, k\right)-E\left(n_{2} \mid s_{2}^{i}=k_{2}, k\right)=\frac{1}{8 \epsilon^{2}}\left[\int_{k_{2}+\epsilon}^{k_{1}+2 \epsilon}\left(2 \epsilon+k_{1}-\theta\right) d \theta-\int_{k_{2}-\epsilon}^{k_{1}}\left(\epsilon-k_{2}+\theta\right) d \theta\right]$.

Integrating this last expression shows that $E\left(n_{2} \mid s_{2}^{i}=k_{1}+\epsilon, k\right)-E\left(n_{2} \mid s_{2}^{i}=\right.$ $\left.k_{2}, k\right)=0$. Q.E.D.

\section{Appendix 3: Iterative Elimination of Domi- nated Strategies}

Let $\left(\bar{s}_{1}^{0}, \bar{s}_{2}^{0}\right)=(\infty, \infty)$. Define $\left(\bar{s}_{1}^{n}, \bar{s}_{2}^{n}\right)$ recursively as the maximal solution to the following system of equations:

$$
\begin{aligned}
& g\left(\bar{s}_{1}^{n},\left(\bar{s}_{1}^{n-1}, \bar{s}_{2}^{n-1}\right)\right)=0, \\
& h\left(\bar{s}_{2}^{n},\left(\bar{s}_{1}^{n-1}, \bar{s}_{2}^{n-1}\right)\right)=0 .
\end{aligned}
$$

LEMMA 5 In the absence of cohort effects, one has $\left(\bar{s}_{1}^{n}, \bar{s}_{2}^{n}\right) \leq\left(\bar{s}_{1}^{n-1}, \bar{s}_{2}^{n-1}\right)$.

Proof: The proof of this lemma proceeds by induction. We start with the following two remarks.

Remark 1: $g\left(\bar{s}_{1}^{n-1},\left(\bar{s}_{1}^{n-2}, \bar{s}_{2}^{n-2}\right)\right) \neq 0$ when $h\left(\bar{s}_{1}^{n-1}-\epsilon,\left(\bar{s}_{1}^{n-2}, \bar{s}_{2}^{n-2}\right)\right) \geq 0$.

Remark 2: If $\gamma=1, h(\cdot)$ is strictly increasing in $s_{2}^{i}$.

Remark 1 follows from the fact that a player who invest at time two irrespective of her first-period error term, strictly gains by investing at time one and saving the 
waiting cost. Remark 2 follows immediately from the fact that $E(n \mid \cdot)$ is weakly increasing in $s_{2}^{i}$. On the basis of both remarks, we know that $g\left(\bar{s}_{1}^{1},(\infty, \infty)\right)$ must be equal to one (and only one) of the following two possibilities:

$$
\begin{gathered}
g\left(\bar{s}_{1}^{1},(\infty, \infty)\right)=\bar{s}_{1}^{1}-1-\frac{\tau}{2}\left(\bar{s}_{1}^{1}+\epsilon-1-\Delta\right), \\
g\left(\bar{s}_{1}^{1},(\infty, \infty)\right)=\bar{s}_{1}^{1}-1 .
\end{gathered}
$$

Both (10) and (11) take into account the fact that $E(n \mid \cdot,(\infty, \infty))=0$. Equation (10) equals zero if $\bar{s}_{1}^{1}=1+\frac{\tau}{2-\tau}(\epsilon-\Delta)<\infty$. Equation (11) equals zero if $\bar{s}_{1}^{1}=1<$ $\infty .{ }^{8}$ Observe also that:

$$
h\left(\bar{s}_{2}^{1},(\infty, \infty)\right)=0 \Leftrightarrow \bar{s}_{2}^{1}=1+\Delta<\infty .
$$

Induction step. Suppose $\left(\bar{s}_{1}^{n-1}, \bar{s}_{2}^{n-1}\right) \leq\left(\bar{s}_{1}^{n-2}, \bar{s}_{2}^{n-2}\right)$, then $\left(\bar{s}_{1}^{n}, \bar{s}_{2}^{n}\right) \leq\left(\bar{s}_{1}^{n-1}, \bar{s}_{2}^{n-1}\right)$.

We first show that $\bar{s}_{1}^{n} \leq \bar{s}_{1}^{n-1}$. We know that:

$$
\begin{gathered}
g\left(\bar{s}_{1}^{n-1},\left(\bar{s}_{1}^{n-2}, \bar{s}_{2}^{n-2}\right)\right)=\bar{s}_{1}^{n-1}+E\left(n \mid \bar{s}_{1}^{n-1},\left(\bar{s}_{1}^{n-2}, \bar{s}_{2}^{n-2}\right)\right)-1 \\
-\frac{\tau}{2} \max \left\{0, h\left(\bar{s}_{1}^{n-1}+\epsilon,\left(\bar{s}_{1}^{n-2}, \bar{s}_{2}^{n-2}\right)\right\}-\frac{\tau}{2} \max \left\{0, h\left(\bar{s}_{1}^{n-1}-\epsilon,\left(\bar{s}_{1}^{n-2}, \bar{s}_{2}^{n-2}\right)\right\} .\right.\right.
\end{gathered}
$$

On the basis of Remark 1 and Remark 2, we can conclude that one (and only one) of the following two cases must prevail:

$$
\begin{aligned}
& g\left(\bar{s}_{1}^{n-1},\left(\bar{s}_{1}^{n-2}, \bar{s}_{2}^{n-2}\right)\right)=\bar{s}_{1}^{n-1}+E\left(n \mid \bar{s}_{1}^{n-1}, \cdot\right)-1-\frac{\tau}{2} h\left(\bar{s}_{1}^{n-1}+\epsilon, \cdot\right), \\
& g\left(\bar{s}_{1}^{n-1},\left(\bar{s}_{1}^{n-2}, \bar{s}_{2}^{n-2}\right)\right)=\bar{s}_{1}^{n-1}+E\left(n \mid \bar{s}_{1}^{n-1}, \cdot\right)-1 .
\end{aligned}
$$

We now show that in case (i) $\bar{s}_{1}^{n} \leq \bar{s}_{1}^{n-1}$. First we show that

$$
g\left(\bar{s}_{1}^{n-1},\left(\bar{s}_{1}^{n-1}, \bar{s}_{2}^{n-1}\right)\right) \geq g\left(\bar{s}_{1}^{n-1},\left(\bar{s}_{1}^{n-2}, \bar{s}_{2}^{n-2}\right)\right)=0 .
$$

To prove inequality (12) we must know how $g\left(\bar{s}_{1}^{n-1},\left(\bar{s}_{1}^{n-1}, \bar{s}_{2}^{n-1}\right)\right)$ looks like. For that purpose, note that:

$$
0 \leq h\left(\bar{s}_{1}^{n-1}+\epsilon,\left(\bar{s}_{1}^{n-2}, \bar{s}_{2}^{n-2}\right)\right) \leq h\left(\bar{s}_{1}^{n-1}+\epsilon,\left(\bar{s}_{1}^{n-1}, \bar{s}_{2}^{n-1}\right)\right),
$$

and that,

$$
h\left(\bar{s}_{1}^{n-1}-\epsilon,\left(\bar{s}_{1}^{n-2}, \bar{s}_{2}^{n-2}\right)\right) \leq h\left(\bar{s}_{1}^{n-1}-\epsilon,\left(\bar{s}_{1}^{n-1}, \bar{s}_{2}^{n-1}\right)\right) .
$$

\footnotetext{
${ }^{8}$ Equation (11) implicitly relies on the assumption that $\bar{s}_{1}^{1}+\epsilon-1=\epsilon \leq \Delta$. Similarly, one can check that equation (10) is valid when $\epsilon>\Delta$.
} 
Both inequalities above follow from the fact that the gain of investing cannot decrease when the other players are more eager to invest. But then it follows that $g\left(\bar{s}_{1}^{n-1},\left(\bar{s}_{1}^{n-1}, \bar{s}_{2}^{n-1}\right)\right)$ must be equal to one (and only one) of the following two possibilities:

(a) $\quad g\left(\bar{s}_{1}^{n-1},\left(\bar{s}_{1}^{n-1}, \bar{s}_{2}^{n-1}\right)\right)=\bar{s}_{1}^{n-1}+E\left(n \mid \bar{s}_{1}^{n-1}, \cdot\right)-1-\frac{\tau}{2} h\left(\bar{s}_{1}^{n-1}+\epsilon, \cdot\right)-\frac{\tau}{2} h\left(\bar{s}_{1}^{n-1}-\epsilon, \cdot\right) \geq \Delta>0$,

(b) $\quad g\left(\bar{s}_{1}^{n-1},\left(\bar{s}_{1}^{n-1}, \bar{s}_{2}^{n-1}\right)\right)=\bar{s}_{1}^{n-1}+E\left(n \mid \bar{s}_{1}^{n-1}, \cdot\right)-1-\frac{\tau}{2} h\left(\bar{s}_{1}^{n-1}+\epsilon, \cdot\right)$.

In subcase (a) it trivially follows that inequality (12) is satisfied. In subcase (b) inequality (12) can be rewritten as

$$
\begin{aligned}
& \frac{1}{2}(1-\tau)\left[E\left(n \mid \bar{s}_{1}^{n-1}+\epsilon,\left(\bar{s}_{1}^{n-1}, \bar{s}_{2}^{n-1}\right)\right)-E\left(n \mid \bar{s}_{1}^{n-1}+\epsilon,\left(\bar{s}_{1}^{n-2}, \bar{s}_{2}^{n-2}\right)\right)\right] \\
& +\frac{1}{2}\left[E\left(n \mid \bar{s}_{1}^{n-1}-\epsilon,\left(\bar{s}_{1}^{n-1}, \bar{s}_{2}^{n-1}\right)\right)-E\left(n \mid \bar{s}_{1}^{n-1}-\epsilon,\left(\bar{s}_{1}^{n-2}, \bar{s}_{2}^{n-2}\right)\right)\right] \geq 0
\end{aligned}
$$

which is obviously satisfied $(E(n \mid \cdot)$ cannot decrease when players become more eager to invest).

We now show that inequality (12) also holds in case (ii). In case (ii), $g\left(\bar{s}_{1}^{n-1},\left(\bar{s}_{1}^{n-1}, \bar{s}_{2}^{n-1}\right)\right)$ can be equal to any one of the following three possibilities:

$$
\begin{aligned}
& \text { (a') } g\left(\bar{s}_{1}^{n-1},\left(\bar{s}_{1}^{n-1}, \bar{s}_{2}^{n-1}\right)\right)=\bar{s}_{1}^{n-1}+E\left(n \mid \bar{s}_{1}^{n-1}, \cdot\right)-1-\frac{\tau}{2} h\left(\bar{s}_{1}^{n-1}+\epsilon, \cdot\right)-\frac{\tau}{2} h\left(\bar{s}_{1}^{n-1}-\epsilon, \cdot\right), \\
& \text { (b') } g\left(\bar{s}_{1}^{n-1},\left(\bar{s}_{1}^{n-1}, \bar{s}_{2}^{n-1}\right)\right)=\bar{s}_{1}^{n-1}+E\left(n \mid \bar{s}_{1}^{n-1}, \cdot\right)-1-\frac{\tau}{2} h\left(\bar{s}_{1}^{n-1}+\epsilon, \cdot\right) \\
& \text { (c') } g\left(\bar{s}_{1}^{n-1},\left(\bar{s}_{1}^{n-1}, \bar{s}_{2}^{n-1}\right)\right)=\bar{s}_{1}^{n-1}+E\left(n \mid \bar{s}_{1}^{n-1}, \cdot\right)-1 .
\end{aligned}
$$

In subcase (a') inequality (12) is trivially satisfied. In subcase (b'), inequality (12) can be rewritten as

$$
E\left(n \mid \bar{s}_{1}^{n-1},\left(\bar{s}_{1}^{n-2}, \bar{s}_{2}^{n-2}\right)\right) \leq E\left(n \mid \bar{s}_{1}^{n-1},\left(\bar{s}_{1}^{n-1}, \bar{s}_{2}^{n-1}\right)\right)-\frac{\tau}{2} h\left(\bar{s}_{1}^{n-1}+\epsilon,\left(\bar{s}_{1}^{n-1}, \bar{s}_{2}^{n-1}\right)\right) .
$$

Note that the rhs of this last inequality is decreasing in $\tau$. Setting $\tau=1$, we can rewrite this last inequality as:

$$
\begin{gathered}
E\left(n \mid \bar{s}_{1}^{n-1},\left(\bar{s}_{1}^{n-2}, \bar{s}_{2}^{n-2}\right)\right) \leq \frac{1}{2} E\left(n \mid s_{2}^{i}=\bar{s}_{1}^{n-1}-\epsilon,\left(\bar{s}_{1}^{n-1}, \bar{s}_{2}^{n-1}\right)\right) \\
-\frac{1}{2}\left(\bar{s}_{1}^{n-1}+\epsilon\right)+\frac{1}{2}(1+\Delta) .
\end{gathered}
$$


Note that the rhs of the inequality above is increasing in $\Delta$. In case (ii), $h\left(\bar{s}_{1}^{n-1}+\right.$ $\left.\epsilon,\left(\bar{s}_{1}^{n-2}, \bar{s}_{2}^{n-2}\right)\right) \leq 0$, which can be rewritten as

$$
\bar{s}_{1}^{n-1}+\epsilon+E\left(n \mid \bar{s}_{1}^{n-1}+\epsilon,\left(\bar{s}_{1}^{n-2}, \bar{s}_{2}^{n-2}\right)\right)-1 \leq \Delta .
$$

Substituting $\Delta$ in (13) by its minimal value given in (14) and after rearranging terms, we can rewrite equation (12) as

$$
E\left(n \mid \bar{s}_{1}^{n-1}-\epsilon,\left(\bar{s}_{1}^{n-2}, \bar{s}_{2}^{n-2}\right)\right) \leq E\left(n \mid \bar{s}_{1}^{n-1}-\epsilon,\left(\bar{s}_{1}^{n-1}, \bar{s}_{2}^{n-1}\right)\right) .
$$

Above we have already shown that this inequality holds. In subcase (c') inequality (12) boils down to

$$
E\left(n \mid s_{1}^{i}=\bar{s}_{1}^{n-1},\left(\bar{s}_{1}^{n-1}, \bar{s}_{2}^{n-1}\right)\right) \geq E\left(n \mid s_{1}^{i}=\bar{s}_{1}^{n-1},\left(\bar{s}_{1}^{n-2}, \bar{s}_{2}^{n-2}\right)\right),
$$

which is obviously satisfied. As $g\left(\bar{s}_{1}^{n-1},\left(\bar{s}_{1}^{n-1}, \bar{s}_{2}^{n-1}\right)\right) \geq g\left(\bar{s}_{1}^{n-1},\left(\bar{s}_{1}^{n-2}, \bar{s}_{2}^{n-2}\right)\right)=0$ and as it is a unique best response to immediately invest for all $s_{1}^{i}>\bar{s}_{1}^{n-1}$, it follows that $s_{1}^{n} \leq s_{1}^{n-1}$.

We are left to show that $\bar{s}_{2}^{n} \leq \bar{s}_{2}^{n-1}$. First, observe that

$$
0 \leq h\left(\bar{s}_{2}^{n-1},\left(\bar{s}_{1}^{n-1}, \bar{s}_{2}^{n-1}\right)\right) \Leftrightarrow E\left(n \mid \bar{s}_{2}^{n-1},\left(\bar{s}_{1}^{n-2}, \bar{s}_{2}^{n-2}\right)\right) \leq E\left(n \mid \bar{s}_{2}^{n-1},\left(\bar{s}_{1}^{n-1}, \bar{s}_{2}^{n-1}\right)\right),
$$

which is obviously satisfied. Second, as $0 \leq h\left(\bar{s}_{2}^{n-1},\left(\bar{s}_{1}^{n-1}, \bar{s}_{2}^{n-1}\right)\right)$ and as $h(\cdot)$ is strictly increasing (and continuous) in its first argument, it follows that $\bar{s}_{2}^{n} \leq \bar{s}_{2}^{n-1}$. Q.E.D.

Let $\left(\underline{s}_{1}^{0}, \underline{s}_{2}^{0}\right)=(-\infty,-\infty)$. Define $\left(\underline{s}_{1}^{n}, \underline{s}_{2}^{n}\right)$ recursively as minimum values satisfying the following system of equations:

$$
\begin{aligned}
& g\left(\underline{s}_{1}^{n},\left(\underline{s}_{1}^{n-1}, \underline{s}_{2}^{n-1}\right)\right)=0, \\
& h\left(\underline{s}_{2}^{n},\left(\underline{s}_{1}^{n-1}, \underline{s}_{2}^{n-1}\right)\right)=0 .
\end{aligned}
$$

LEMMA 6 In the absence of a cohort effect, one has $\left(\underline{s}_{1}^{n}, \underline{s}_{2}^{n}\right) \leq\left(\underline{s}_{1}^{n-1}, \underline{s}_{2}^{n-1}\right)$.

Proof: The proof of this lemma mirrors the one we outlined in Lemma (5). On the basis of Remark 1 and Remark 2, we know that $g\left(\underline{s}_{1}^{1},(-\infty,-\infty)\right)$ must be equal to one of the following two possibilities:

$$
g\left(\underline{s}_{1}^{1},(-\infty,-\infty)\right)=\underline{s}_{1}^{1}-\frac{\tau}{2}\left(\underline{s}_{1}^{1}+\epsilon-\Delta\right),
$$




$$
g\left(\underline{s}_{1}^{1},(-\infty,-\infty)\right)=\underline{s}_{1}^{1} .
$$

Both (15) and (16) take into account the fact that $E(n \mid \cdot,(-\infty,-\infty))=1$. Equation (15) equals zero if $\underline{s}_{1}^{1}=\frac{\tau}{2-\tau}(\epsilon-\tau)>-\infty$. Equation (16) equals zero if $\underline{s}_{1}^{1}=0 .{ }^{9}$ Observe also that $h\left(s_{2}^{i},(-\infty,-\infty)\right)=0$ if $s_{2}^{i}=\Delta>-\infty$.

Induction step: Suppose $\left(\underline{s}_{1}^{n-1}, \underline{s}_{2}^{n-1}\right) \geq\left(\underline{s}_{1}^{n-2}, \underline{s}_{2}^{n-2}\right)$, then $\left(\underline{s}_{1}^{n}, \underline{s}_{2}^{n}\right) \geq\left(\underline{s}_{1}^{n-1}, \underline{s}_{2}^{n-1}\right)$.

We first show that $\bar{s}_{1}^{n} \geq \bar{s}_{1}^{n-1}$. On the basis of Remark 1, Remark 2 , and continuity of $g($ ), we know that one (and only one) of the following two cases must prevail:

(i) $\quad g\left(\underline{s}_{1}^{n-1},\left(\underline{s}_{1}^{n-2}, \underline{s}_{2}^{n-2}\right)\right)=\underline{s}_{1}^{n-1}+E\left(n \mid \underline{s}_{1}^{n-1}, \cdot\right)-1-\frac{\tau}{2} h\left(\underline{s}_{1}^{n-1}+\epsilon, \cdot\right)$,

(ii) $\quad g\left(\underline{s}_{1}^{n-1},\left(\underline{s}_{1}^{n-2}, \underline{s}_{2}^{n-2}\right)\right)=\underline{s}_{1}^{n-1}+E\left(n \mid \underline{s}_{1}^{n-1}, \cdot\right)-1$.

We first tackle the case where the expression for $g\left(\underline{s}_{1}^{n-1},\left(\underline{s}_{1}^{n-2}, \underline{s}_{2}^{n-2}\right)\right)$ is given by equation (i). We prove that

$$
g\left(\underline{s}_{1}^{n-1},\left(\underline{s}_{1}^{n-1}, \underline{s}_{2}^{n-1}\right)\right) \leq g\left(\underline{s}_{1}^{n-1},\left(\underline{s}_{1}^{n-2}, \underline{s}_{2}^{n-2}\right)\right) .
$$

$g\left(\underline{s}_{1}^{n-1},\left(\underline{s}_{1}^{n-1}, \underline{s}_{2}^{n-1}\right)\right)$ must be equal to one (and only one) of the following three possibilities:

(a) $\quad g\left(\underline{s}_{1}^{n-1},\left(\underline{s}_{1}^{n-1}, \underline{s}_{2}^{n-1}\right)\right)=\underline{s}_{1}^{n-1}+E\left(n \mid \underline{s}_{1}^{n-1}, \cdot\right)-1-\frac{\tau}{2} h\left(\underline{s}_{1}^{n-1}+\epsilon, \cdot\right)-\frac{\tau}{2} h\left(\underline{s}_{1}^{n-1}-\epsilon, \cdot\right)$,

(b) $g\left(\underline{s}_{1}^{n-1},\left(\underline{s}_{1}^{n-1}, \underline{s}_{2}^{n-1}\right)\right)=\underline{s}_{1}^{n-1}+E\left(n \mid \underline{s}_{1}^{n-1}, \cdot\right)-1-\frac{\tau}{2} h\left(\underline{s}_{1}^{n-1}+\epsilon, \cdot\right)$,

(c) $\quad g\left(\underline{s}_{1}^{n-1},\left(\underline{s}_{1}^{n-1}, \underline{s}_{2}^{n-1}\right)\right)=\underline{s}_{1}^{n-1}+E\left(n \mid \underline{s}_{1}^{n-1}, \cdot\right)-1$.

Case (i) implicitly relies on the assumption that

$$
\underline{s}_{1}^{n-1}-\epsilon+E\left(n \mid s_{2}^{i}=\underline{s}_{1}^{n-1}-\epsilon,\left(\underline{s}_{1}^{n-2}, \underline{s}_{2}^{n-2}\right)\right)-1<\Delta .
$$

Subcase (a) relies on the assumption that

$$
\underline{s}_{1}^{n-1}-\epsilon+E\left(n \mid s_{2}^{i}=\underline{s}_{1}^{n-1}-\epsilon,\left(\underline{s}_{1}^{n-1}, \underline{s}_{2}^{n-1}\right)\right)-1 \geq \Delta .
$$

As $\left(\underline{s}_{1}^{n-1}, \underline{s}_{2}^{n-1}\right) \geq\left(\underline{s}_{1}^{n-2}, \underline{s}_{2}^{n-2}\right)$, it follows that

$$
E\left(n \mid s_{2}^{i}=\underline{s}_{1}^{n-1}-\epsilon,\left(\underline{s}_{1}^{n-2}, \underline{s}_{2}^{n-2}\right)\right) \geq E\left(n \mid s_{2}^{i}=\underline{s}_{1}^{n-1}-\epsilon,\left(\underline{s}_{1}^{n-1}, \underline{s}_{2}^{n-1}\right)\right),
$$

\footnotetext{
${ }^{9}$ As before, equation (15) implicitly relies on the assumption that $\underline{s}_{1}^{1}+\epsilon>\Delta$ or that $\epsilon>\Delta$. Similarly, one can check that equation (16) is valid when $\epsilon \leq \Delta$.
} 
$(E(n \mid \cdot)$ cannot increase when players become less eager to invest). But this implies that (18) and (19) cannot simultaneously be satisfied and thus that subcase (a) is not a valid expression for $g\left(\underline{s}_{1}^{n-1},\left(\underline{s}_{1}^{n-1}, \underline{s}_{2}^{n-1}\right)\right)$. In subcase (b) inequality (17) can be rewritten as (after rearranging terms)

$$
\begin{aligned}
& \frac{1}{2}(1-\tau)\left[E\left(n \mid \underline{s}_{1}^{n-1}+\epsilon,\left(\underline{s}_{1}^{n-1}, \underline{s}_{2}^{n-1}\right)\right)-E\left(n \mid \underline{s}_{1}^{n-1}+\epsilon,\left(\underline{s}_{1}^{n-2}, \underline{s}_{2}^{n-2}\right)\right)\right] \\
& +\frac{1}{2}\left[E\left(n \mid \underline{s}_{1}^{n-1}-\epsilon,\left(\underline{s}_{1}^{n-1}, \underline{s}_{2}^{n-1}\right)\right)-E\left(n \mid \underline{s}_{1}^{n-1}-\epsilon,\left(\underline{s}_{1}^{n-2}, \underline{s}_{2}^{n-2}\right)\right)\right] \leq 0 .
\end{aligned}
$$

As $E\left(n \mid s_{2}^{1},\left(\underline{s}_{1}^{n-1}, \underline{s}_{2}^{n-1}\right)\right) \leq E\left(n \mid s_{2}^{1},\left(\underline{s}_{1}^{n-2}, \underline{s}_{2}^{n-2}\right)\right)$, this implies that the inequality above is respected. In subcase (c) the inequality (17) can be written as

$$
E\left(n \mid s_{1}^{n-1},\left(\underline{s}_{1}^{n-2}, \underline{s}_{2}^{n-2}\right)\right)-\frac{\tau}{2}\left(\underline{s}_{1}^{n-1}+\epsilon+E\left(n \mid \underline{s}_{1}^{n-1}+\epsilon,\left(\underline{s}_{1}^{n-2}, \underline{s}_{2}^{n-2}\right)\right)-1-\Delta\right) \geq E\left(n \mid \underline{s}_{1}^{n-1},\left(\underline{s}_{1}^{n-1}, \underline{s}_{2}^{n-1}\right)\right)
$$

Note that the lhs is decreasing in $\tau$ and increasing in $\Delta$. In subcase (c) we know that

$$
\underline{s}_{1}^{n-1}+\epsilon+E\left(n \mid \underline{s}_{1}^{n-1}+\epsilon,\left(\underline{s}_{1}^{n-1}, \underline{s}_{2}^{n-1}\right)\right)-1 \leq \Delta .
$$

Setting $\tau=1$ and replacing $\Delta$ by its minimal value given in our last inequality yields

$$
E\left(n \mid \underline{s}_{1}^{n-1}-\epsilon,\left(\underline{s}_{1}^{n-2}, \underline{s}_{2}^{n-2}\right)\right) \geq E\left(n \mid \underline{s}_{1}^{n-1}-\epsilon,\left(\underline{s}_{1}^{n-1}, \underline{s}_{2}^{n-1}\right)\right),
$$

which is obviously satisfied.

Using a reasoning similar to the one we outlined in subcase (i)(a), the reader can check that in case (ii), (a) and (b) are no valid expressions for $g\left(\underline{s}_{1}^{n-1},\left(\underline{s}_{1}^{n-2}, \underline{s}_{2}^{n-2}\right)\right)$. In subcase (ii)(c) inequality (17) boils down to

$$
E\left(n \mid \underline{s}_{1}^{n-1},\left(\underline{s}_{1}^{n-1}, \underline{s}_{2}^{n-1}\right)\right) \leq E\left(n \mid \underline{s}_{1}^{n-1},\left(\underline{s}_{1}^{n-2}, \underline{s}_{2}^{n-2}\right)\right),
$$

which is obviously satisfied. As $g\left(\underline{s}_{1}^{n-1},\left(\underline{s}_{1}^{n-1}, \underline{s}_{2}^{n-1}\right)\right) \leq g\left(\underline{s}_{1}^{n-1},\left(\underline{s}_{1}^{n-2}, \underline{s}_{2}^{n-2}\right)\right)$ and as it is a unique best response not to invest for all $s_{1}^{i}<\underline{s}_{1}^{n-1}$, it follows that $\underline{s}_{1}^{n} \geq \underline{s}_{1}^{n-1}$.

We are left to show that $\underline{s}_{2}^{n} \geq \underline{s}_{2}^{n-1}$. Observe that

$$
0 \geq h\left(\underline{s}_{2}^{n-1},\left(\underline{s}_{1}^{n-1}, \underline{s}_{2}^{n-1}\right)\right) \Leftrightarrow E\left(n \mid \underline{s}_{2}^{n-1},\left(\underline{s}_{1}^{n-2}, \underline{s}_{2}^{n-2}\right)\right) \geq E\left(n \mid \underline{s}_{2}^{n-1},\left(\underline{s}_{1}^{n-1}, \underline{s}_{2}^{n-1}\right)\right),
$$

which is obviously satisfied. As $0 \geq h\left(\underline{s}_{2}^{n-1},\left(\underline{s}_{1}^{n-1}, \underline{s}_{2}^{n-1}\right)\right)$ and as $h(\cdot)$ is strictly increasing (and continuous) in its first argument, it follows that $\underline{s}_{2}^{n} \geq \bar{s}_{2}^{n-1}$. Q.E.D. 
Proof of Proposition (5): From Lemma (5), we know that the sequence $\left(\bar{s}_{1}^{n}, \bar{s}_{2}^{n}\right)$ is non increasing, $<<(\infty, \infty) \forall n>1$, and bounded from below by the Nash equilibrium (which must survive iterative elimination of dominated strategies). Similarly, from Lemma (6), we know that the sequence $\left(\underline{s}_{1}^{n}, \underline{s}_{2}^{n}\right)$ is non decreasing, $>>(-\infty,-\infty) \forall n>1$, and bounded from above by the Nash equilibrium. Hence, both sequences converges to some limit $\left(k_{1}, k_{2}\right)$, which satisfies

$$
\begin{aligned}
& g\left(k_{1},\left(k_{1}, k_{2}\right)\right)=0, \\
& h\left(k_{2},\left(k_{1}, k_{2}\right)\right)=0 .
\end{aligned}
$$

Thus, both sequences converges to the unique symmetric switching equilibrium strategy profile. Q.E.D. 


\section{References}

Carlsson, H. and E. van Damme "Global Games and Equilibrium Selection," Econometrica, September 1993, 989-1018

Chamley, C. "Coordinating Regime Switches," Quartely Journal of Economics, August 1999, 869-905

Corsetti, G., A. Dasgupta, S. Morris, and H. S. Shin "Does One Soros Make a Difference? A Theory of Currency Crises with Large and Small Traders," mimeo, August 2000

Dasgupta, A. "Coordination, Learning and Delay," mimeo, December 2001

Diamond, D. and P. Dybvig "Bank Runs, Deposit Insurance, and Liquidity," Journal of Political Economy, March 1983, 91, 401-419

Dönges, J. and F. Heinemann "Competition for Order Flow as a Coordination Game," Unpublished Working Paper, June 2000

Frankel, D. and A. Pauzner "Resolving Indeterminacy in Dynamic Settings: The Role of Shocks," Quartely Journal of Economics, 115, 2000, 285-304

Goldstein, I. and A. Pauzner "Demand Deposit Contracts and the Probability of Bank Runs," mimeo, April 2000

Heinemann, F. "Globale Spiele: Vermeidung multipler Gleichgewichte durch private Informationen," mimeo, 2001

Morris, S. and H. S. Shin "Global Games: Theory and Applications," mimeo, 2001

Morris, S. and H. S. Shin "The CNBC Effect: Welfare Effects of Public Information," mimeo, 2001

Morris, S. and H. S. Shin "A Theory of the Onset of Currency Attacks," in, Asian Financial Crisis: Causes, Contagion and Consequences, Agenon, Vines and Weiber Eds., Cambridge University Press

O'Brien, P. "Analysts' Forecasts as Earnings Expectations," Journal of Accounting and Economics, January 1988, 10, 53-83

Obstfeld, M. "Models of Currency Crises with Self-Fulfilling features," European Economic Review, 40, 1996, 1037-1047

Postlewaite, A. and X. Vives "Bank Runs as an Equilibrium Phenomenon," Journal of Political Economy, 1987, 95, 485-491

Rochet, J.-C. and X. Vives "Coordination Failures and the Lender of Last resort: Was Bagehot Right After All?" mimeo, IAE, 2000

Rubinstein, A. "The Electronic Mail Game: Strategic Behavior Under "Almost Common Knowledge"," American Economic Review, 1989, 79, 385-391 


\section{Appendix 4 (for referees only)}

Appendix 4 proves that $g(\cdot)>0$, if and only if $s_{1}^{i}>k_{1}$. We start by defining:

$$
\begin{aligned}
l_{1}\left(s_{1}^{i}, k\right) \equiv & s_{1}^{i}+E\left(n_{1}+\alpha n_{2} \mid s_{1}^{i}, k\right)-1, \\
l_{2}\left(s_{1}^{i}, k\right) \equiv & s_{1}^{i}+E\left(n_{1}+\alpha n_{2} \mid s_{1}^{i}, k\right)-1-\frac{\tau}{2}\left[s_{1}^{i}+\epsilon+E\left(\gamma n_{1}+n_{2} \mid s_{2}^{i}=s_{1}^{i}+\epsilon, k\right)-1-\Delta\right], \\
l_{3}\left(s_{1}^{i}, k\right) \equiv & s_{1}^{i}+E\left(n_{1}+\alpha n_{2} \mid s_{1}^{i}, k\right)-1-\frac{\tau}{2}\left[s_{1}^{i}-\epsilon+E\left(\gamma n_{1}+n_{2} \mid s_{2}^{i}=s_{1}^{i}-\epsilon, k\right)-1-\Delta\right], \\
l_{4}\left(s_{1}^{i}, k\right) \equiv & s_{1}^{i}+E\left(n_{1}+\alpha n_{2} \mid s_{1}^{i}, k\right)-1-\frac{\tau}{2}\left[s_{1}^{i}+\epsilon+E\left(\gamma n_{1}+n_{2} \mid s_{2}^{i}=s_{1}^{i}+\epsilon, k\right)-1-\Delta\right] \\
& \quad-\frac{\tau}{2}\left[s_{1}^{i}-\epsilon+E\left(\gamma n_{1}+n_{2} \mid s_{2}^{i}=s_{1}^{i}-\epsilon, k\right)-1-\Delta\right] .
\end{aligned}
$$

Note that $g(\cdot)=\min \left\{l_{1}(\cdot), l_{2}(\cdot), l_{3}(\cdot), l_{4}(\cdot)\right\}$.

LEMma 7 For any $k$ that solves equations (4) and (5) and for which $k_{2} \in\left(k_{1}-\right.$ $\left.\epsilon, k_{1}+\epsilon\right)$, one has $g\left(s_{1}^{i}, k\right)<0, \forall s_{1}^{i} \in\left[k_{2}-\epsilon, k_{1}\right)$.

Proof: In this case $k_{2}-\epsilon<s_{1}^{i}<k_{1}$. Hence, $k_{2}<s_{1}^{i}+\epsilon<k_{1}+\epsilon$ and $s_{1}^{i}-\epsilon<k_{1}-\epsilon<$ $k_{2}$. From lemma (h-fn), we thus know that $h\left(s_{1}^{i}+\epsilon, k\right)>0$ and $h\left(s_{1}^{i}-\epsilon, k\right)<0$. Therefore in this case $g(\cdot)=l_{2}(\cdot)$. By Leibnitz's rule, one has

$$
\begin{gathered}
\frac{\partial l_{2}(\cdot)}{\partial s_{1}^{i}}=1+\frac{1}{4 \epsilon}\left[n_{1}\left(\theta=s_{1}^{i}+2 \epsilon, k\right)-n_{1}\left(\theta=s_{1}^{i}-2 \epsilon, k\right)\right. \\
\left.+\alpha n_{2}\left(\theta=s_{1}^{i}+2 \epsilon, k\right)-\alpha n_{2}\left(\theta=s_{1}^{i}-2 \epsilon, k\right)\right]-\frac{\tau}{2}-\frac{\tau}{4 \epsilon}\left[\gamma n_{1}\left(\theta=s_{1}^{i}+2 \epsilon, k\right)\right. \\
\left.-\gamma n_{1}\left(\theta=s_{1}^{i}, k\right)+n_{2}\left(\theta=s_{1}^{i}+2 \epsilon, k\right)-n_{2}\left(\theta=s_{1}^{i}, k\right)\right] .
\end{gathered}
$$

Using Appendix 1, we can evaluate this derivative at the point where $s_{1}^{i}=k_{1}$, and, after some rearranging, we get

$$
\frac{\partial l_{2}\left(s_{1}^{i}=k_{1}, k\right)}{\partial s_{1}^{i}}=1-\frac{\tau}{2}+\frac{1}{8 \epsilon}(2-\tau \gamma)+\frac{k_{1}+\epsilon-k_{2}}{16 \epsilon^{2}} \tau,
$$

which is positive for $\gamma, \tau \leq 1$ and $k_{2}<k_{1}+\epsilon$. Note also that

$$
\frac{\partial^{2} l_{2}\left(s_{1}^{i}=k_{1}, k\right)}{\partial s_{1}^{i^{2}}}=\frac{1-\alpha}{16 \epsilon^{2}}+\frac{\tau}{8 \epsilon^{2}}>0 .
$$

Hence, since the second derivative is a positive constant, we know that (i) $l_{2}(\cdot)$ is a symmetric convex function on the domain $\left[k_{2}-\epsilon, k_{1}\right]$. Therefore, (ii) $l_{2}(\cdot)$ possesses 
at most two roots. Since $k$ solves the equations (4) and (5), $l_{2}\left(s_{1}^{i}=k_{1}, k\right)=0$ and because $\frac{\partial l_{2}\left(s_{1}^{i}=k_{1}, k\right)}{\partial s_{1}^{i}}>0$, (iii) the upper root equals $k_{1}$. As $l_{2}(\cdot)$ is a symmetric and convex function it follows that $g\left(s_{1}^{i}=k_{2}-\epsilon, k\right)<0$ if and only if the absolute value of the slope of $g(\cdot)$ evaluated at the point $k_{2}-\epsilon$ is lower than the slope of $g(\cdot)$ evaluated at $k_{1}$. More formally, $g\left(s_{1}^{i}=k_{2}-\epsilon, k\right)<0$ if and only if

$$
\frac{\partial l_{2}\left(s_{1}^{i}=k_{1}, k\right)}{\partial s_{1}^{i}}+\frac{\partial l_{2}\left(s_{1}^{i}=k_{2}-\epsilon, k\right)}{\partial s_{1}^{i}}>0 .
$$

Using Appendix 1 to evaluate (20) at $s_{1}^{i}=k_{2}-\epsilon$ and rewriting yields

$$
\begin{aligned}
\frac{\partial l_{2}\left(s_{1}^{i}=k_{2}-\epsilon, k\right)}{\partial s_{1}^{i}}= & 1-\frac{\tau}{2}+\frac{1}{4 \epsilon}\left(\frac{k_{2}+3 \epsilon-k_{1}}{4 \epsilon}+\alpha \frac{k_{1}+\epsilon-k_{2}}{4 \epsilon}\right) \\
& -\frac{\gamma \tau}{8 \epsilon}-\frac{k_{1}+\epsilon-k_{2}}{16 \epsilon^{2}} \tau .
\end{aligned}
$$

Observe that $\frac{\partial l_{2}\left(s_{1}^{i}=k_{1}, k\right)}{\partial s_{1}^{i}}$ is independent of $\alpha$ and that $\frac{\partial l_{2}\left(s_{1}^{i}=k_{2}-\epsilon, k\right)}{\partial s_{1}^{i}}$ is increasing in $\alpha$. Thus substituting (21) and (23) into inequality (22) and setting $\alpha=0$ yields the following sufficient condition for $g\left(s_{1}^{i}=k_{2}-\epsilon, k\right)<0$,

$$
2\left(1-\frac{\tau}{2}\right)+\frac{1}{16 \epsilon^{2}}\left(k_{2}+3 \epsilon-k_{1}\right)+\frac{1}{4 \epsilon}-\frac{\gamma \tau}{4 \epsilon}>0 .
$$

As $k_{2}+3 \epsilon>k_{1}$ and $\gamma, \tau \leq 1$, this condition is always satisfied. As $l_{2}(\cdot)$ is a convex function and $l_{2}\left(s_{1}^{i}=k_{2}-\epsilon, k\right)<0$ and $l_{2}\left(s_{1}^{i}=k_{1}, k\right)=0$, it follows that $l_{2}(\cdot)<0$ $\forall s_{1}^{i} \in\left[k_{2}-\epsilon, k_{1}\right)$. Q.E.D.

To show that $g(\cdot)<0 \forall s_{1}^{i}<k_{2}-\epsilon$, it is useful to note that:

LEMMA 8 For any $k$ that solves equations (4) and (5) and for which $k_{2} \in\left(k_{1}-\right.$ $\left.\epsilon, k_{1}+\epsilon\right)$, one has $E\left(n_{1} \mid s_{1}^{i}, k\right)+\alpha E\left(n_{2} \mid s_{1}^{i}, k\right)$ is weakly increasing in $s_{1}^{i}$.

Proof: Using Appendix 1, it is easy to verify that $n_{1}(\theta, k)+n_{2}(\theta, k)$ is (weakly) increasing in $\theta$. Suppose that $n_{1}(\cdot)+\alpha n_{2}(\cdot)$ is strictly decreasing in $\theta$ for some $\theta$. This requires that $0<\frac{\partial n_{1}(\cdot)}{\partial \theta}<-\alpha \frac{\partial n_{2}(\cdot)}{\partial \theta}$. Since $\alpha \leq 1$, this implies thus that $\frac{\partial n_{1}(\cdot)}{\partial \theta}<-\frac{\partial n_{2}(\cdot)}{\partial \theta}$ and thus that $n_{1}(\cdot)+n_{2}(\cdot)$ decreases in $\theta$, a contradiction. We conclude that $n_{1}(\cdot)+n_{2}(\cdot)$ is weakly increasing in $\theta$. Because

$$
E\left(n_{1}+\alpha n_{2} \mid s_{1}^{i}, k\right)=\frac{1}{4 \epsilon} \int_{s_{1}^{i}-2 \epsilon}^{s_{1}^{i}+2 \epsilon}\left[n_{1}(\theta, k)+\alpha n_{2}(\theta, k)\right] d \theta
$$


Leibnitz's rule implies that

$\frac{\partial E\left(n_{1}+\alpha n_{2} \mid s_{1}^{i}, k\right)}{\partial s_{1}^{i}}=\left[n_{1}\left(s_{1}^{i}+\epsilon, k\right)+\alpha n_{2}\left(s_{1}^{i}+\epsilon, k\right)\right]-\left[n_{1}\left(s_{1}^{i}-\epsilon, k\right)+\alpha n_{2}\left(s_{1}^{i}-\epsilon, k\right)\right] \geq 0$,

were the last inequality follows from the fact that $n_{1}(\cdot)+\alpha n_{2}(\cdot)$ is weakly increasing in $\theta$. Thus, $E\left(n_{1}+\alpha n_{2} \mid s_{1}^{i}, k\right)$ is weakly increasing in $s_{1}^{i}$. Q.E.D.

LEMMA 9 For any $k$ that solves equations (4) and (5) and for which $k_{2} \in\left(k_{1}-\right.$ $\left.\epsilon, k_{1}+\epsilon\right)$, one has $g\left(s_{1}^{i}, k\right)<0, \forall s_{1}^{i}<k_{2}-\epsilon$.

Proof: As $s_{1}^{i}<k_{2}-\epsilon$ it follows that $s_{1}^{i}-\epsilon<s_{1}^{i}+\epsilon<k_{2}$. From Lemma (4), we thus know that in this case $g(\cdot)=l_{1}(\cdot)$. Observe that

$$
\frac{\partial l_{1}\left(s_{1}^{i}, k\right)}{\partial s_{1}^{i}}=1+\frac{\partial E\left(n_{1}+\alpha n_{2} \mid s_{1}^{i}, k\right)}{\partial s_{1}^{i}} .
$$

From Lemma (8), we know that $E\left(n_{1}+\alpha n_{2} \mid s_{1}^{i}, k\right)$ is weakly increasing in $s_{1}^{i}$. Hence $\frac{\partial l_{1}\left(s_{1}^{i}, k\right)}{\partial s_{1}^{i}}>0$. From Lemma $(7)$, we also know that $l_{1}\left(s_{1}^{i}=k_{2}-\epsilon, k\right)=l_{2}\left(s_{1}^{i}=\right.$ $\left.k_{2}-\epsilon, k\right)<0$. Hence, $l_{1}(\cdot)<0 \forall s_{1}^{i}<k_{2}-\epsilon$. Q.E.D.

LEMma 10 For any $k$ that solves equations (4) and (5) and for which $k_{2} \in\left(k_{1}-\right.$ $\left.\epsilon, k_{1}+\epsilon\right)$, one has $l_{1}\left(s_{1}^{i}, k\right)>0, \forall s_{1}^{i} \geq k_{1}$.

Proof: By definition $l_{2}\left(s_{1}^{i}=k_{1}, k\right)=l_{1}\left(s_{1}^{i}=k_{1}, k\right)-\frac{\tau}{2} h\left(k_{1}+\epsilon, k\right)$. Since $k$ solves equations (4) and $(5), l_{2}\left(s_{1}^{i}=k_{1}, k\right)=0$. Furthermore, by Lemma (4), $h\left(k_{1}+\epsilon, k\right)>0$. Therefore, $l_{1}\left(s_{1}^{i}=k_{1}, k\right)>0$. Recall that

$$
\frac{\partial l_{1}\left(s_{1}^{i}, k\right)}{\partial s_{1}^{i}}=1+\frac{\partial E\left(n_{1}+\alpha n_{2} \mid s_{1}^{i}, k\right)}{\partial s_{1}^{i}} .
$$

From Lemma (8), we know that $E\left(n_{1}+\alpha n_{2} \mid s_{1}^{i}, k\right)$ is weakly increasing in $s_{1}^{i}$. Hence $\frac{\partial l_{1}\left(s_{1}^{i}, k\right)}{\partial s_{1}^{i}}>0$. Thus, $l_{1}\left(s_{1}^{i}, k\right)$ is strictly increasing in $s_{1}^{i}$ and since we showed above that $l_{1}\left(s_{1}^{i}=k_{1}, k\right)>0$, this proves our lemma. Q.E.D.

LEMMA 11 For any $k$ that solves equations (4) and (5) and for which $k_{2} \in\left(k_{1}-\right.$ $\left.\epsilon, k_{1}+\epsilon\right)$, one has $g\left(s_{1}^{i}, k\right)>0, \forall s_{1}^{i} \in\left(k_{1}, k_{2}+\epsilon\right]$. 
Proof: By Lemma 10, $l_{1}(\cdot)>0$. Since in this case $k_{1}<s_{1}^{i} \leq k_{2}+\epsilon$, Lemma (4) implies that $h\left(s_{1}^{i}-\epsilon, k\right) \leq 0$. Thus, $g(\cdot)$ cannot equal $l_{3}(\cdot)$ or $l_{4}(\cdot)$. We are left to verify that $l_{2}(\cdot)>0$. Using Lemma $(3)$, we can rewrite $l_{2}(\cdot)$ as

$$
\begin{gathered}
l_{2}(\cdot)=\left(1-\frac{\tau}{2}\right)\left(s_{1}^{i}-1\right)+\frac{\tau}{2}(\Delta-\epsilon)+\frac{1-\tau \gamma}{2} E\left(n_{1} \mid s_{2}^{i}=s_{1}^{i}+\epsilon, k\right) \\
+\frac{\alpha-\tau}{2} E\left(n_{2} \mid s_{2}^{i}=s_{1}^{i}+\epsilon, k\right)+\frac{1}{2} E\left(n_{1} \mid s_{2}^{i}=s_{1}^{i}-\epsilon, k\right)+\frac{\alpha}{2} E\left(n_{2} \mid s_{2}^{i}=s_{1}^{i}-\epsilon, k\right) .
\end{gathered}
$$

By Leibnitz's rule, one has

$$
\begin{aligned}
\frac{\partial l_{2}(\cdot)}{\partial s_{1}^{i}}= & \left(1-\frac{\tau}{2}\right)+\frac{1-\tau \gamma}{4 \epsilon}\left\{n_{1}\left(s_{1}^{i}+2 \epsilon, k\right)-n_{1}\left(s_{1}^{i}, k\right)\right\}+\frac{\alpha-\tau}{4 \epsilon}\left\{n_{2}\left(s_{1}^{i}+2 \epsilon, k\right)-n_{2}\left(s_{1}^{i}, k\right)\right\} \\
& +\frac{1}{4 \epsilon}\left\{n_{1}\left(s_{1}^{i}, k\right)-n_{1}\left(s_{1}^{i}-2 \epsilon, k\right)\right\}+\frac{\alpha}{4 \epsilon}\left\{n_{2}\left(s_{1}^{i}, k\right)-n_{2}\left(s_{1}^{i}-2 \epsilon, k\right)\right\} .
\end{aligned}
$$

Using $k_{1}<s_{1}^{i}<k_{2}+\epsilon, k_{1}+2 \epsilon<s_{1}^{i}+2 \epsilon, k_{1}-2 \epsilon<s_{1}^{i}-2 \epsilon<k_{2}-\epsilon$ and the $n_{i}(\cdot, k)$ functions derived in Appendix 1, one can rewrite the above equation as

$$
\frac{\partial l_{2}(\cdot)}{\partial s_{1}^{i}}=\left(1-\frac{\tau}{2}\right)+\frac{1-\tau \gamma}{4 \epsilon}+\frac{\tau \gamma}{4 \epsilon}\left(\frac{1}{2}+\frac{s_{1}^{i}-k_{1}}{4 \epsilon}\right)+\frac{\tau}{4 \epsilon}\left(\frac{k_{1}+\epsilon-k_{2}}{4 \epsilon}\right)-\frac{1}{4 \epsilon}\left(\frac{s_{1}^{i}-k_{1}}{4 \epsilon}\right) .
$$

Hence,

$$
\frac{\partial^{2} l_{2}(\cdot)}{\left(\partial s_{1}^{i}\right)^{2}}=\frac{1}{16 \epsilon^{2}}(\tau \gamma-1) \leq 0
$$

Thus, $l_{2}(\cdot)$ is concave in $s_{1}^{i}$ over the domain $\left(k_{1}, k_{2}+\epsilon\right]$. Hence, a sufficient condition for $\frac{\partial l_{2}(\cdot)}{\partial s_{1}^{i}}>0$ for all $s_{1}^{i} \in\left(k_{1}, k_{2}+\epsilon\right]$ is that $\frac{\partial l_{2}\left(s_{1}^{i}=k_{2}+\epsilon, k\right)}{\partial s_{1}^{i}}>0$, where

$$
\frac{\partial l_{2}\left(s_{1}^{i}=k_{2}+\epsilon, k\right)}{\partial s_{1}^{i}}=\left(1-\frac{\tau}{2}\right)+\frac{1-\tau \gamma}{4 \epsilon}-\frac{1+\tau-\tau \gamma}{16 \epsilon^{2}}\left(k_{2}-k_{1}\right)+\frac{1}{16 \epsilon}(3 \tau \gamma+\tau-1) .
$$

Since $1+\tau>\tau \gamma$, this function is decreasing in $k_{2}-k_{1}$. Substituting $k_{2}-k_{1}=\epsilon$ on the right hand side of the above expression and rewriting shows that

$$
\frac{\partial l_{2}\left(s_{1}^{i}=k_{2}+\epsilon, k\right)}{\partial s_{1}^{i}} \geq\left(1-\frac{\tau}{2}\right)+\frac{1}{8 \epsilon}>0 .
$$

Hence, we have shown that $l_{2}(\cdot)>0$ for all $s_{1}^{i} \in\left(k_{1}, k_{2}+\epsilon\right]$. Q.E.D.

LEMMA 12 For any $k$ that solves equations (4) and (5) and for which $k_{2} \in\left(k_{1}-\right.$ $\left.\epsilon, k_{1}+\epsilon\right)$, one has $g\left(s_{1}^{i}, k\right)>0, \forall s_{1}^{i} \in\left(k_{2}+\epsilon, k_{1}+2 \epsilon\right]$. 
Proof: In this case $k_{2}+\epsilon<s_{1}^{i} \leq k_{1}+2 \epsilon$. Hence, $k_{2}<s_{1}^{i}-\epsilon \leq k_{1}+\epsilon$, and Lemma (4) implies that $h\left(s_{1}^{i}-\epsilon, k\right)>0$. Therefore, $g(\cdot)$ cannot be equal to $l_{2}(\cdot)$ or $l_{1}(\cdot)$. We are left to establish that $l_{3}(\cdot)>0$ and $l_{4}(\cdot)>0$ for all $s_{1}^{i} \in\left(k_{2}+\epsilon, k_{1}+2 \epsilon\right]$. By Lemma (3), one has

$$
l_{4}\left(s_{1}^{i}, k\right)=(1-\tau \gamma) E\left(n_{1} \mid s_{1}^{i}, k\right)+(\alpha-\tau) E\left(n_{2} \mid s_{1}^{i}, k\right)+\tau \Delta+\left(s_{1}^{i}-1\right)(1-\tau) .
$$

From Leibnitz's rule follows that:

$$
\begin{aligned}
& \frac{\partial l_{4}\left(s_{1}^{i}, k\right)}{\partial s_{1}^{i}}=\frac{1-\tau \gamma}{4 \epsilon}\left[n_{1}\left(s_{1}^{i}+2 \epsilon, k\right)-n_{1}\left(s_{1}^{i}-2 \epsilon, k\right)\right] \\
& +\frac{\alpha-\tau}{4 \epsilon}\left[n_{2}\left(s_{1}^{i}+2 \epsilon, k\right)-n_{2}\left(s_{1}^{i}-2 \epsilon, k\right)\right]+(1-\tau) .
\end{aligned}
$$

Using Appendix 1, we can rewrite this equation as

$$
\begin{gathered}
\frac{\partial l_{4}\left(s_{1}^{i}, k\right)}{\partial s_{1}^{i}}=\frac{1-\tau \gamma}{16 \epsilon^{2}}\left[4 \epsilon-s_{1}^{i}+k_{1}\right]+\frac{\alpha-\tau}{16 \epsilon^{2}}\left[k_{2}+\epsilon-s_{1}^{i}\right]+(1-\tau) \\
\geq \frac{1-\tau}{16 \epsilon^{2}}\left[\left(k_{1}+2 \epsilon-s_{1}^{i}\right)+\left(k_{2}+3 \epsilon-s_{1}^{i}\right)\right]+(1-\tau)>0,
\end{gathered}
$$

where the first inequality follows from the facts that $k_{1}+4 \epsilon>s_{1}^{i}, \gamma \leq 1, k_{2}+\epsilon<s_{1}^{i}$ and $\alpha \leq 1$. The second inequality follows from $k_{1}+2 \epsilon \geq s_{1}^{i}$ and $k_{2}+3 \epsilon>k_{1}+2 \epsilon \geq$ $s_{1}^{i}$. We are left to consider $l_{3}(\cdot)$. By Lemma (3), one has

$$
\begin{aligned}
l_{3}\left(s_{1}^{i}, k\right)= & \left(1-\frac{\tau}{2}\right)\left(s_{1}^{i}-1\right)+\frac{\tau}{2}(\Delta+\epsilon)+\frac{1}{2} E\left(n_{1} \mid s_{2}^{i}=s_{1}^{i}+\epsilon, k\right)+\frac{\alpha}{2} E\left(n_{2} \mid s_{2}^{i}=s_{1}^{i}+\epsilon, k\right) \\
& +\frac{1-\tau \gamma}{2} E\left(n_{1} \mid s_{2}^{i}=s_{1}^{i}-\epsilon, k\right)+\frac{\alpha-\tau}{2} E\left(n_{2} \mid s_{2}^{i}=s_{1}^{i}-\epsilon, k\right) .
\end{aligned}
$$

By Leibnitz's rule, one has

$$
\begin{gathered}
\frac{\partial l_{3}\left(s_{1}^{i}, k\right)}{\partial s_{1}^{i}}=\left(1-\frac{\tau}{2}\right)+\frac{1}{4 \epsilon}\left[n_{1}\left(s_{1}^{i}+2 \epsilon, k\right)-n_{1}\left(s_{1}^{i}, k\right)\right]+\frac{\alpha}{4 \epsilon}\left[n_{2}\left(s_{1}^{i}+2 \epsilon, k\right)-n_{2}\left(s_{1}^{i}, k\right)\right] \\
+\frac{1-\tau \gamma}{4 \epsilon}\left[n_{1}\left(s_{1}^{i}, k\right)-n_{1}\left(s_{1}^{i}-2 \epsilon, k\right)\right]+\frac{\alpha-\tau}{4 \epsilon}\left[n_{2}\left(s_{1}^{i}, k\right)-n_{2}\left(s_{1}^{i}-2 \epsilon, k\right)\right]
\end{gathered}
$$

Using Appendix 1, $k_{2}+\epsilon<s_{1}^{i} \leq k_{1}+2 \epsilon$, and rewriting (using the fact that $n_{2}\left(s_{1}^{i}, k\right)=n_{1}\left(s_{1}^{i}+2 \epsilon, k\right)-n_{1}\left(s_{1}^{i}, k\right)$ in this case) yields

$$
\frac{\partial l_{3}\left(s_{1}^{i}, k\right)}{\partial s_{1}^{i}}=\left(1-\frac{\tau}{2}\right)+\frac{1-\tau}{4 \epsilon}\left(\frac{k_{1}+2 \epsilon-s_{1}^{i}}{4 \epsilon}\right)+\frac{1-\tau \gamma}{8 \epsilon}-\frac{\alpha-\tau}{4 \epsilon}\left(\frac{s_{1}^{i}-\left(k_{2}+\epsilon\right)}{4 \epsilon}\right)
$$




$$
\begin{aligned}
\geq\left(1-\frac{\tau}{2}\right)+ & \frac{1-\tau \gamma}{8 \epsilon}+\frac{1-\tau}{4 \epsilon}\left(\frac{\left(k_{1}+2 \epsilon\right)+\left(k_{2}+\epsilon\right)-2 s_{1}^{i}}{4 \epsilon}\right) \\
& \geq\left(1-\frac{\tau}{2}\right)+\frac{1-\tau \gamma}{8 \epsilon}+\frac{1-\tau}{8 \epsilon} \geq 0
\end{aligned}
$$

where the first inequality follows from the facts that $\alpha \leq 1$ and $s_{1}^{i}>k_{2}+\epsilon$, the second inequality from the fact that $\left(k_{1}+2 \epsilon\right)+\left(k_{2}+\epsilon\right)-2 s_{1}^{i}<2 \epsilon$, and the last inequality from the fact that $\gamma \leq 1$. Hence, $l_{3}(\cdot)$ is strictly increasing in the range $\left(k_{2}+\epsilon, k_{1}+2 \epsilon\right]$. Since, by Lemmas (4) and (10), $l_{3}\left(s_{1}^{i}=k_{2}+\epsilon, k\right)=l_{1}\left(s_{1}^{i}=\right.$ $\left.k_{2}+\epsilon, k\right)>0$, this implies that $l_{3}(\cdot)>0$ for all $s_{1}^{i} \in\left(k_{2}+\epsilon, k_{1}+2 \epsilon\right]$. Q.E.D.

LEMMA 13 For any $k$ that solves equations (4) and (5) and for which $k_{2} \in\left(k_{1}-\right.$ $\left.\epsilon, k_{1}+\epsilon\right)$, one has $g\left(s_{1}^{i}, k\right)>0, \forall s_{1}^{i} \in\left(k_{1}+2 \epsilon, k_{1}+4 \epsilon\right]$.

Proof: By Lemma (10), $l_{1}(\cdot)>0$. Note that for all $k_{1}+2 \epsilon \leq s_{1}^{i} \leq k_{1}+2 \epsilon$, $E\left(n_{1} \mid s_{2}^{i}=s_{1}^{i}+\epsilon, k\right)=1$ and $E\left(n_{2} \mid s_{2}^{i}=s_{1}^{i}+\epsilon, k\right)=0$. Hence, using Lemma (3), we can rewrite $l_{2}(\cdot)$ as

$l_{2}(\cdot)=\left(1-\frac{\tau}{2}\right)\left(s_{1}^{i}-1\right)+\frac{\tau}{2}(\Delta-\epsilon)+\frac{1-\tau \gamma}{2}+\frac{1}{2} E\left(n_{1} \mid s_{2}^{i}=s_{1}^{i}-\epsilon, k\right)+\frac{\alpha}{2} E\left(n_{2} \mid s_{2}^{i}=s_{1}^{i}-\epsilon, k\right)$.

Using Leibnitz's rule gives

$\frac{\partial l_{2}\left(s_{1}^{i}, k\right)}{\partial s_{1}^{i}}=\left(1-\frac{\tau}{2}\right)+\frac{1}{4 \epsilon}\left[n_{1}\left(s_{1}^{i}, k\right)+\alpha n_{2}\left(s_{1}^{i}, k\right)-\left(n_{1}\left(s_{1}^{i}-2 \epsilon, k\right)+\alpha n_{2}\left(s_{1}^{i}-2 \epsilon, k\right)\right)\right] \geq 0$,

since $n_{1}(\theta, k)+\alpha n_{2}(\theta, k)$ is nondecreasing. Since Lemma (12) states that $g\left(s_{1}^{i}=\right.$ $\left.k_{1}+2 \epsilon, k\right)>0$ and thus also $l_{2}\left(s_{1}^{i}=k_{1}+2 \epsilon, k\right)>0$, this implies that $l_{2}(\cdot)>0$ $\forall s_{1}^{i} \in\left(k_{1}+2 \epsilon, k_{1}+4 \epsilon\right]$.

Using $E\left(n_{1} \mid s_{2}^{i}=s_{1}^{i}+\epsilon, k\right)=1, E\left(n_{2} \mid s_{2}^{i}=s_{1}^{i}+\epsilon, k\right)=0$ and Lemma (3) to rewrite $l_{3}(\cdot)$, gives

$$
\begin{gathered}
l_{3}\left(s_{1}^{i}, k\right)=\left(1-\frac{\tau}{2}\right)\left(s_{1}^{i}-1\right)+\frac{\tau}{2}(\Delta-\epsilon)+\frac{1}{2} \\
+\frac{1-\tau \gamma}{2} E\left(n_{1} \mid s_{2}^{i}=s_{1}^{i}-\epsilon, k\right)+\frac{\alpha-\tau}{2} E\left(n_{2} \mid s_{2}^{i}=s_{1}^{i}-\epsilon, k\right) .
\end{gathered}
$$

By Leibnitz's rule, one has

$\frac{\partial l_{3}\left(s_{1}^{i}, k\right)}{\partial s_{1}^{i}}=\left(1-\frac{\tau}{2}\right)+\frac{1-\tau \gamma}{4 \epsilon}\left[n_{1}\left(s_{1}^{i}, k\right)-n_{1}\left(s_{1}^{i}-2 \epsilon, k\right)\right]+\frac{\alpha-\tau}{4 \epsilon}\left[n_{2}\left(s_{1}^{i}, k\right)-n_{2}\left(s_{1}^{i}-2 \epsilon, k\right)\right]$.

Since, by Appendix $1, n_{2}\left(s_{1}^{i}, k\right)=0$ in this case and $\gamma, \alpha \leq 1$, the above implies that

$\frac{\partial l_{3}\left(s_{1}^{i}, k\right)}{\partial s_{1}^{i}} \geq\left(1-\frac{\tau}{2}\right)+\frac{1-\tau}{4 \epsilon}\left[n_{1}\left(s_{1}^{i}, k\right)+n_{2}\left(s_{1}^{i}, k\right)-\left(n_{1}\left(s_{1}^{i}-2 \epsilon, k\right)+n_{2}\left(s_{1}^{i}-2 \epsilon, k\right)\right)\right]$, 
which is positive because $n_{1}(\theta, k)+n_{2}(\theta, k)$ is nondecreasing. We conclude that $l_{3}(\cdot)>0 \forall s_{1}^{i} \in\left(k_{1}+2 \epsilon, k_{1}+4 \epsilon\right]$ since it is increasing and $l_{3}\left(s_{1}^{i}=k_{1}+2 \epsilon, k\right)>0$ by Lemma (12).

Recall that by Lemma (3), one has

$$
l_{4}\left(s_{1}^{i}, k\right)=(1-\tau \gamma) E\left(n_{1} \mid s_{1}^{i}, k\right)+(\alpha-\tau) E\left(n_{2} \mid s_{1}^{i}, k\right)+\tau \Delta+\left(s_{1}^{i}-1\right)(1-\tau)
$$

and, by Leibnitz's rule, that

$$
\begin{aligned}
& \frac{\partial l_{4}\left(s_{1}^{i}, k\right)}{\partial s_{1}^{i}}=\frac{1-\tau \gamma}{4 \epsilon}\left[n_{1}\left(s_{1}^{i}+2 \epsilon, k\right)-n_{1}\left(s_{1}^{i}-2 \epsilon, k\right)\right] \\
& +\frac{\alpha-\tau}{4 \epsilon}\left[n_{2}\left(s_{1}^{i}+2 \epsilon, k\right)-n_{2}\left(s_{1}^{i}-2 \epsilon, k\right)\right]+(1-\tau) .
\end{aligned}
$$

Since, by Appendix $1, n_{2}\left(s_{1}^{i}+2 \epsilon, k\right)=0$ in this case and $\gamma, \alpha \leq 1$, one has

$\frac{\partial l_{4}\left(s_{1}^{i}, k\right)}{\partial s_{1}^{i}} \geq \frac{1-\tau}{4 \epsilon}\left[n_{1}\left(s_{1}^{i}+2 \epsilon, k\right)+n_{2}\left(s_{1}^{i}+2 \epsilon, k\right)-\left(n_{1}\left(s_{1}^{i}-2 \epsilon, k\right)+n_{2}\left(s_{1}^{i}-2 \epsilon, k\right)\right)\right]+(1-\tau)$.

As $n_{1}(\theta, k)+n_{2}(\theta, k)$ is nondecreasing, we conclude that $l_{4}(\cdot)>0 \forall s_{1}^{i} \in\left(k_{1}+\right.$ $\left.2 \epsilon, k_{1}+4 \epsilon\right]$ because it is increasing and $l_{4}\left(s_{1}^{i}=k_{1}+2 \epsilon, k\right)>0$ by Lemma (12).

Together with Lemma $(10)$ the above implies that $l_{1}(\cdot), l_{2}(\cdot), l_{3}(\cdot)$ and $l_{4}(\cdot)>0$. Q.E.D.

LEMma 14 For any $k$ that solves equations (4) and (5) and for which $k_{2} \in\left(k_{1}-\right.$ $\left.\epsilon, k_{1}+\epsilon\right)$, one has $g\left(s_{1}^{i}, k\right)>0, \forall s_{1}^{i}>k_{1}+4 \epsilon$.

Proof: By Lemma $(10), l_{1}(\cdot)>0$. The argument for why $l_{3}(\cdot)>0$ and $l_{4}(\cdot)>$ 0 is the same as in the proof of Lemma (13) and thus omitted. Note that if $s_{1}^{i}>k_{1}+4 \epsilon$, we know that $E\left(n_{1} \mid s_{2}^{i}=s_{1}^{i}+\epsilon, k\right)=E\left(n_{1} \mid s_{2}^{i}=s_{1}^{i}-\epsilon, k\right)=1$ and $E\left(n_{2} \mid s_{2}^{i}=s_{1}^{i}+\epsilon, k\right)=E\left(n_{2} \mid s_{2}^{i}=s_{1}^{i}-\epsilon, k\right)=0$. Hence, in this case

$$
l_{2}(\cdot)=\left(1-\frac{\tau}{2}\right)\left(s_{1}^{i}-1\right)+\frac{\tau}{2}(\Delta-\epsilon)+\frac{1-\tau \gamma}{2}+\frac{1}{2} .
$$

Since $\frac{\partial l_{2}(\cdot)}{\partial s_{1}^{i}}>0$ and by Lemma (13) $l_{2}\left(s_{1}^{i}=k_{1}+4 \epsilon, k\right)>0, l_{2}(\cdot)>0$ for all $s_{1}^{i}>k_{1}+4 \epsilon$. Q.E.D. 